Source rock potential and geochemical characterization (total organic carbon, rockeval, and gas chromatogram mass spectrography of extracted organic matter) of the Tenneco Oil Company OCS Y-0943-1 (Aurora No. 1) well as determined from unwashed cuttings $\left(6,960^{\prime}-14,190 '\right)$.

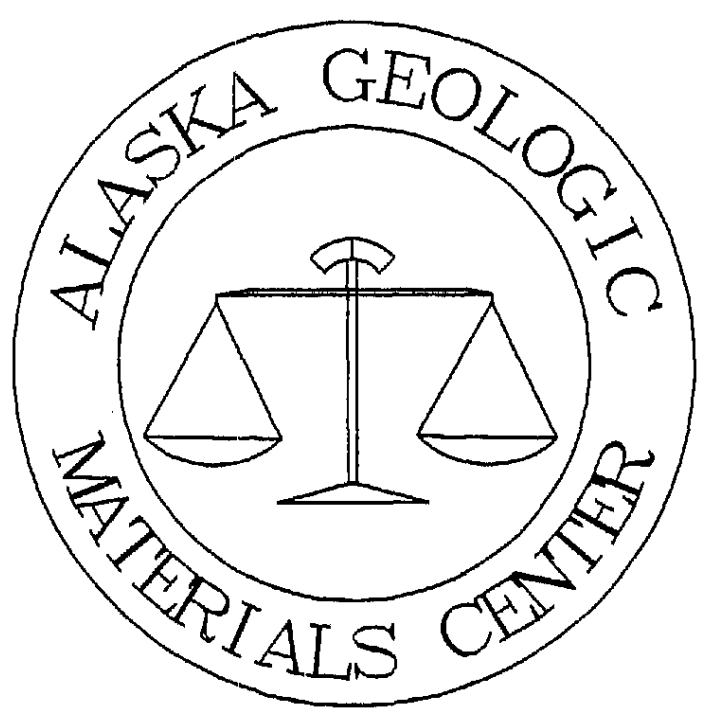


TENNECO AURORA \#1 (API $\$ 551410000400$ ) GEOCHEMICAL ANALYSIS

- performed by by DGSI, Houston, July 1992

\begin{tabular}{|c|c|c|c|c|c|c|c|c|c|c|c|c|c|c|c|}
\hline $\begin{array}{c}\text { Sample } \\
\text { Code }\end{array}$ & $\begin{array}{c}\text { DGS } \\
\text { Sample * }\end{array}$ & $\begin{array}{c}\text { Samplo } \\
\text { Type }\end{array}$ & $\begin{array}{c}\text { Sample } \\
\text { Depth }\end{array}$ & $\begin{array}{c}\text { Approx. } \\
\text { Ago }\end{array}$ & Toc & s1 & s2 & s3 & $\max$ & $\mathrm{HI}$ & 여 & $52 / 53$ & $\mathbf{P}$ & $\begin{array}{c}\text { Source } \\
\text { Potential }\end{array}$ & $\begin{array}{l}\text { Thermal } \\
\text { Maturity }\end{array}$ \\
\hline -TA_. & $92 / 2284-1$ & _cuttings & - $69660-7560$ & _Mid_Eocene_ & $\underline{0.96}$ & -0.02 & -0.31. & 1.04 & -439 & -32 & -108 & 0.3 & $\ldots$ & poor ${ }_{\text {Lqas }}$ & mature \\
\hline TA 2 _ & $\underline{9} 2 / 2284-2$ & _cuttings & $13620-13710$ & Early Eocene & $1.2 \underline{9}$ & -0.35 & 0.93 & 2.05 & -437. & 72 & -159 & 0.45 & $\ldots .27$ & poor ${ }_{\text {_qas }}$ & mature \\
\hline TA 3 & $92 / 2284-3$ & cultings & $14010-14190$ & Paleocene & 1.33 & 0.18 & 0.79 & 0.67 & 448 & 59 & 50 & 1.18 & 0.19 & poor, gas & mature \\
\hline
\end{tabular}




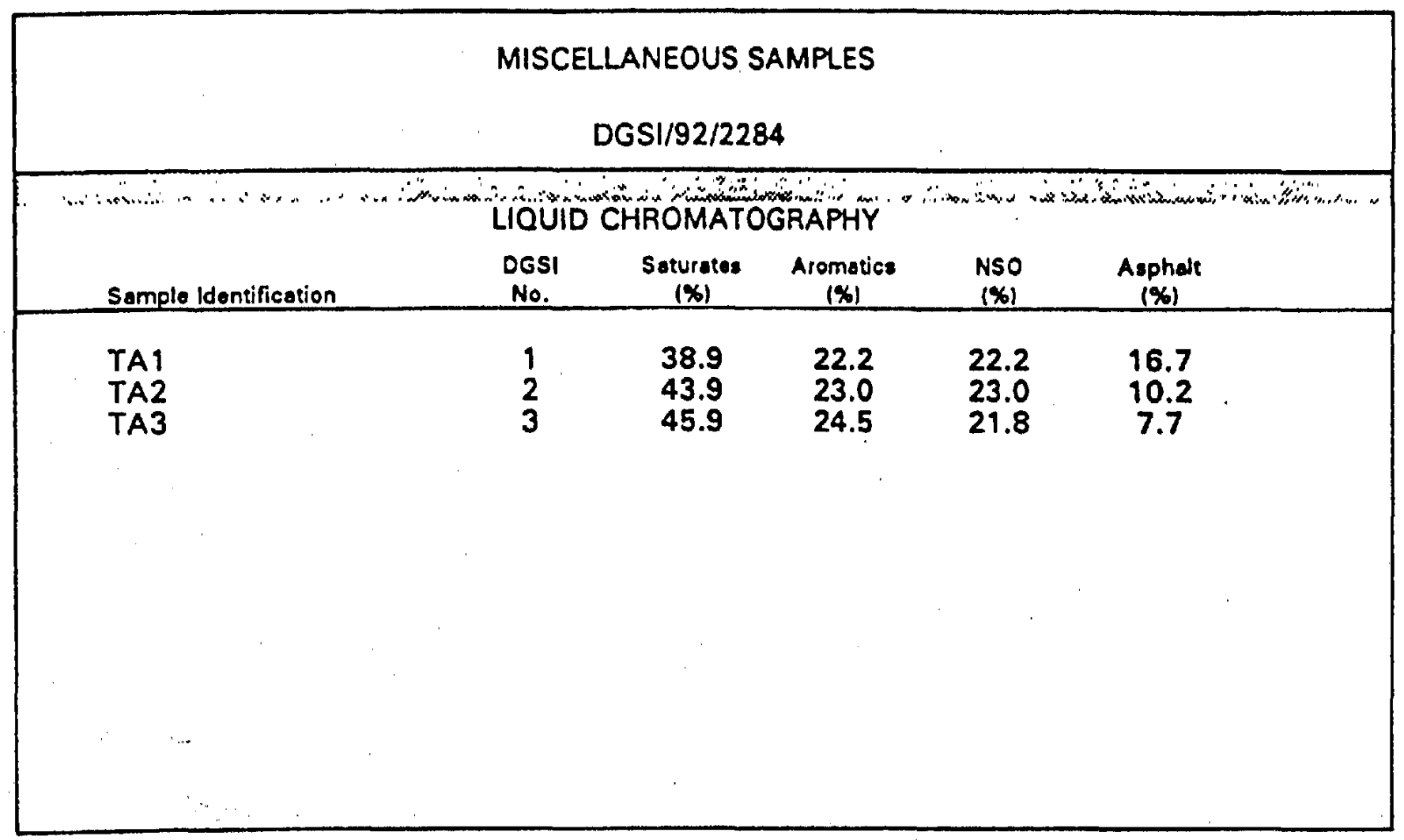

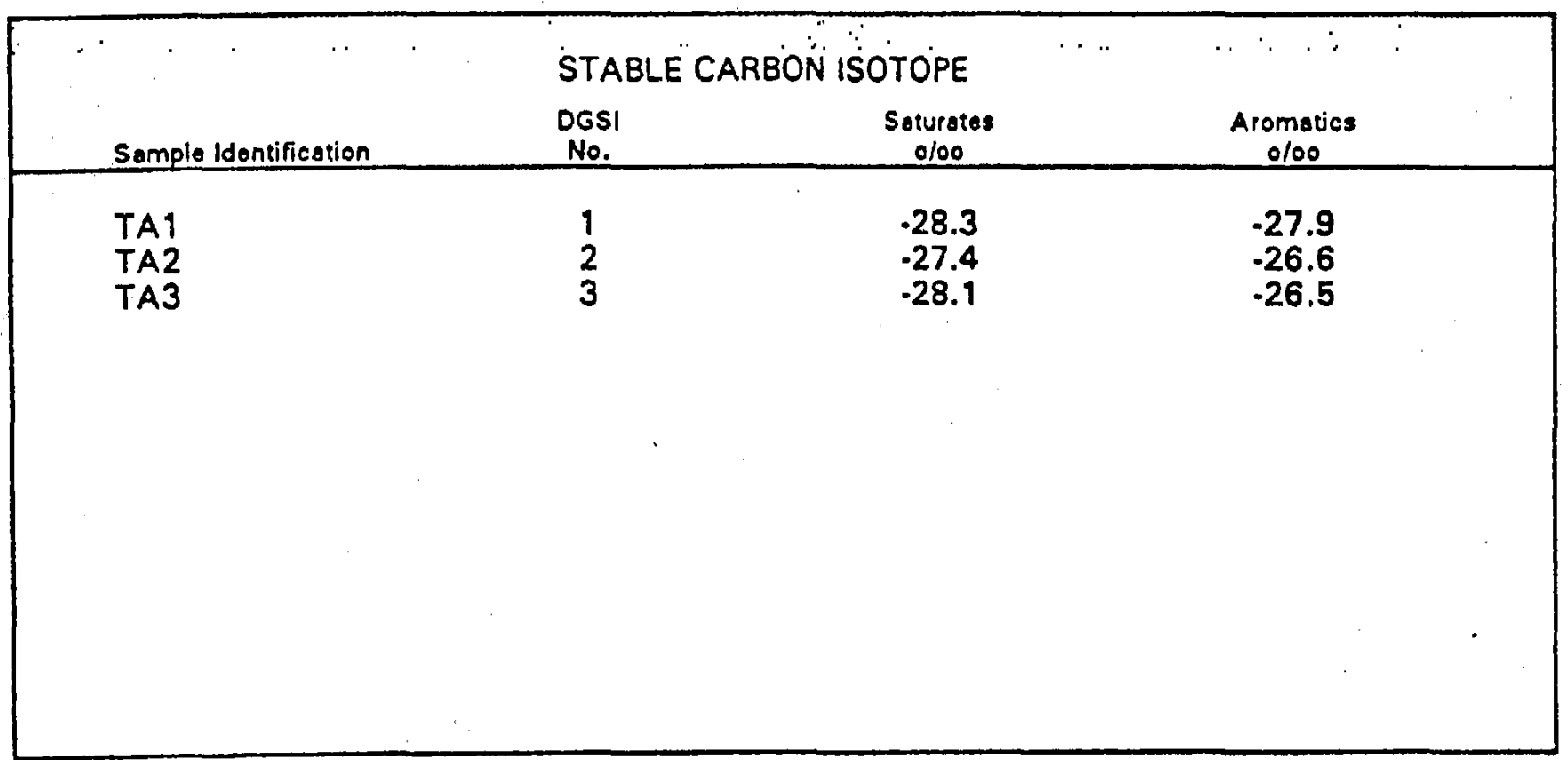




\section{MISCELLANEOUS SAMPLES}

DGSI/92/2284

\section{EXTRACT CALCULATIONS}

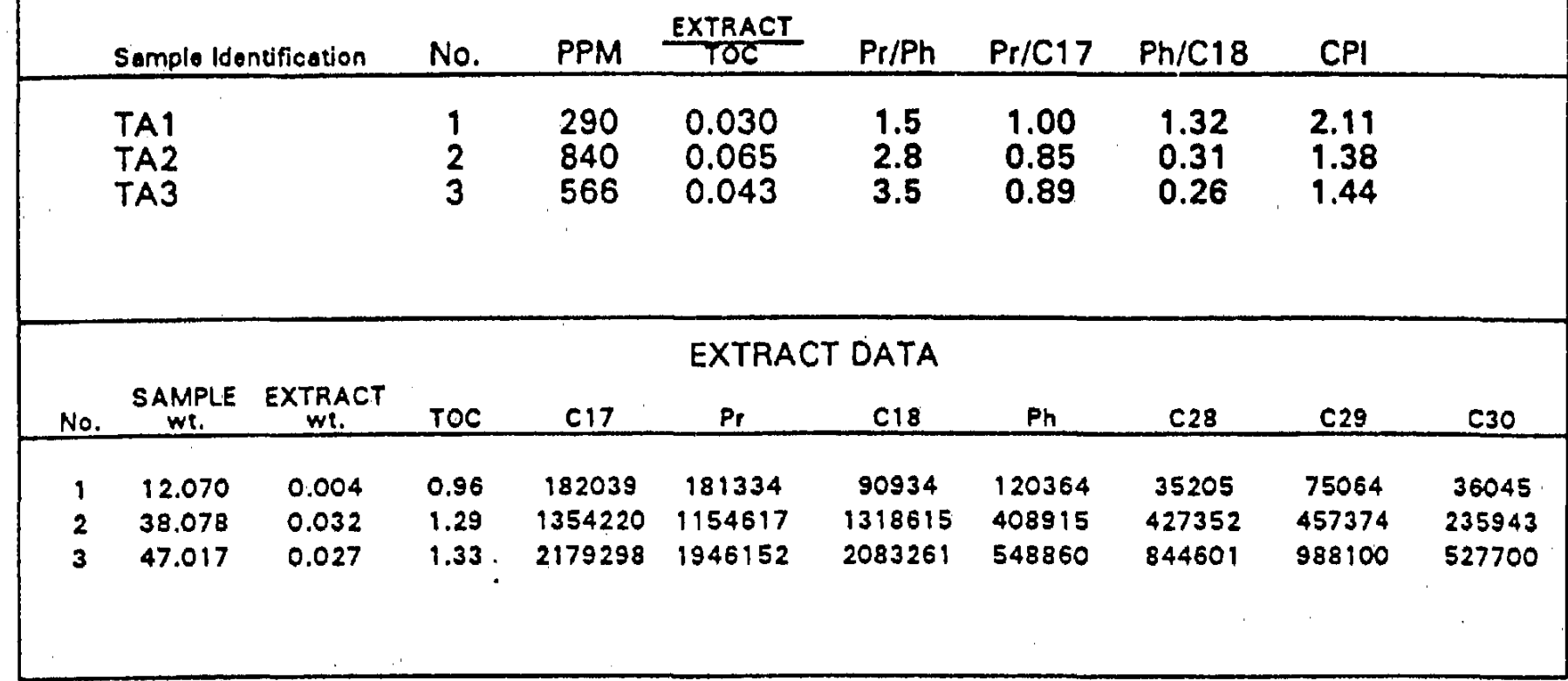

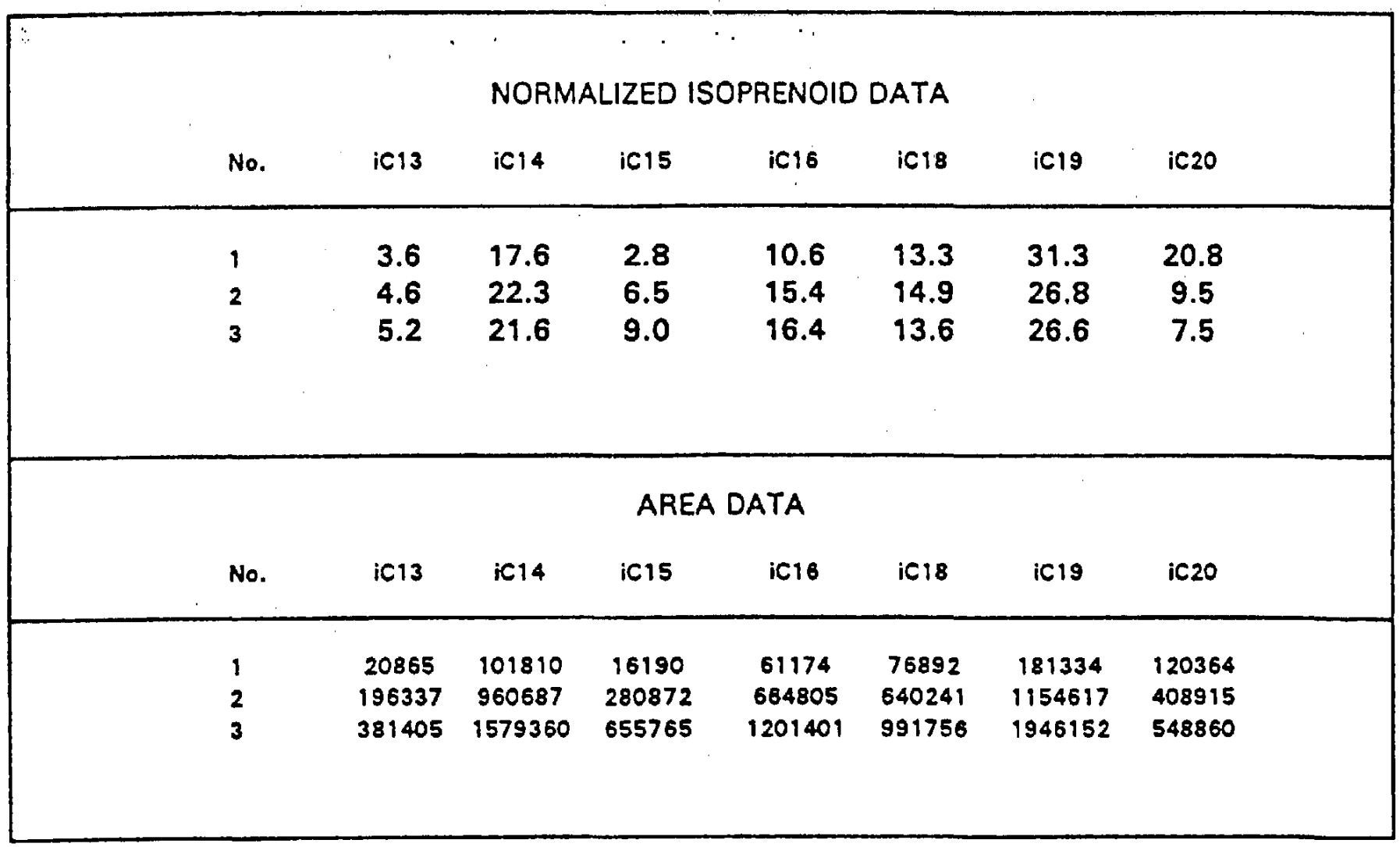




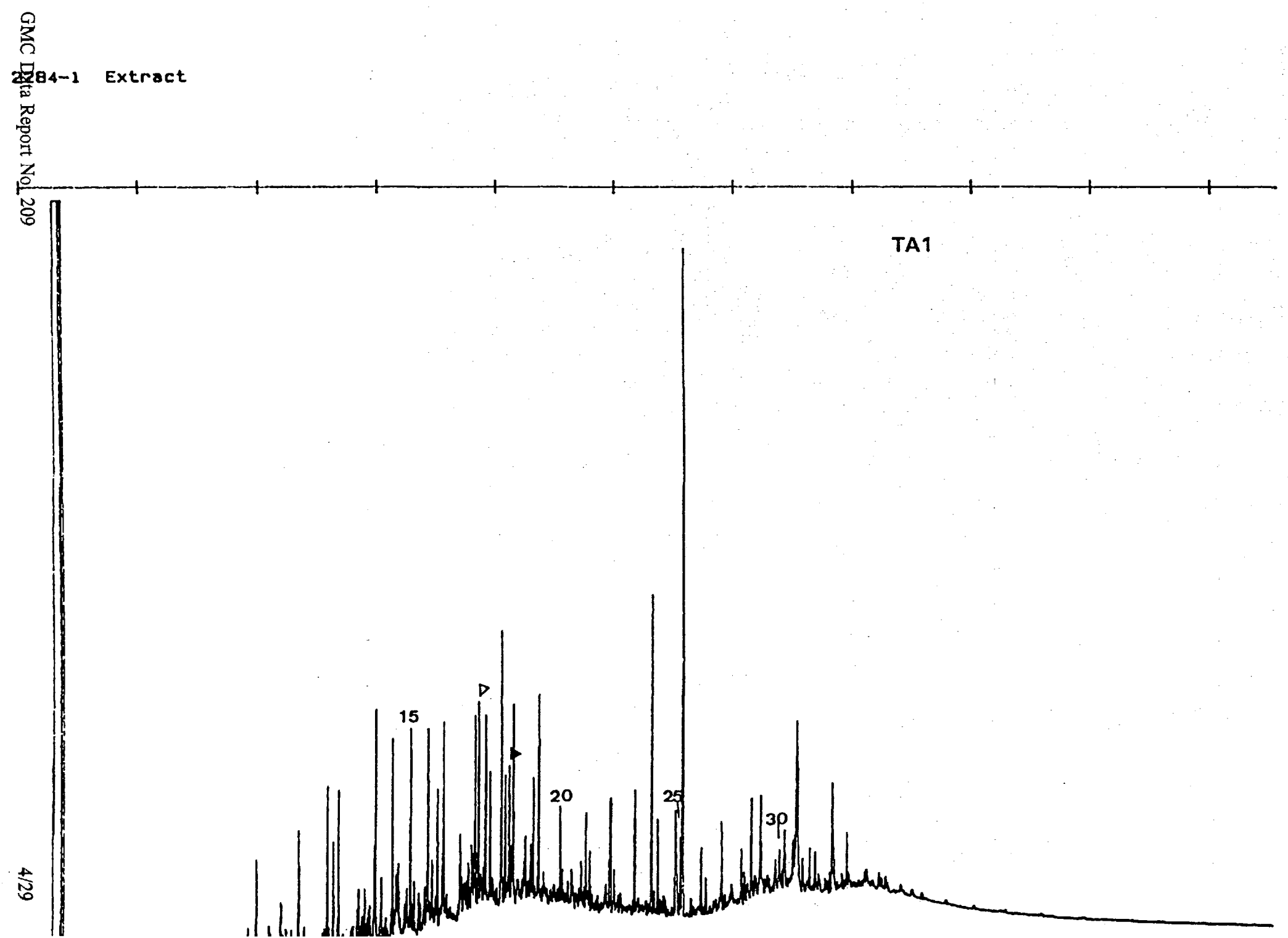




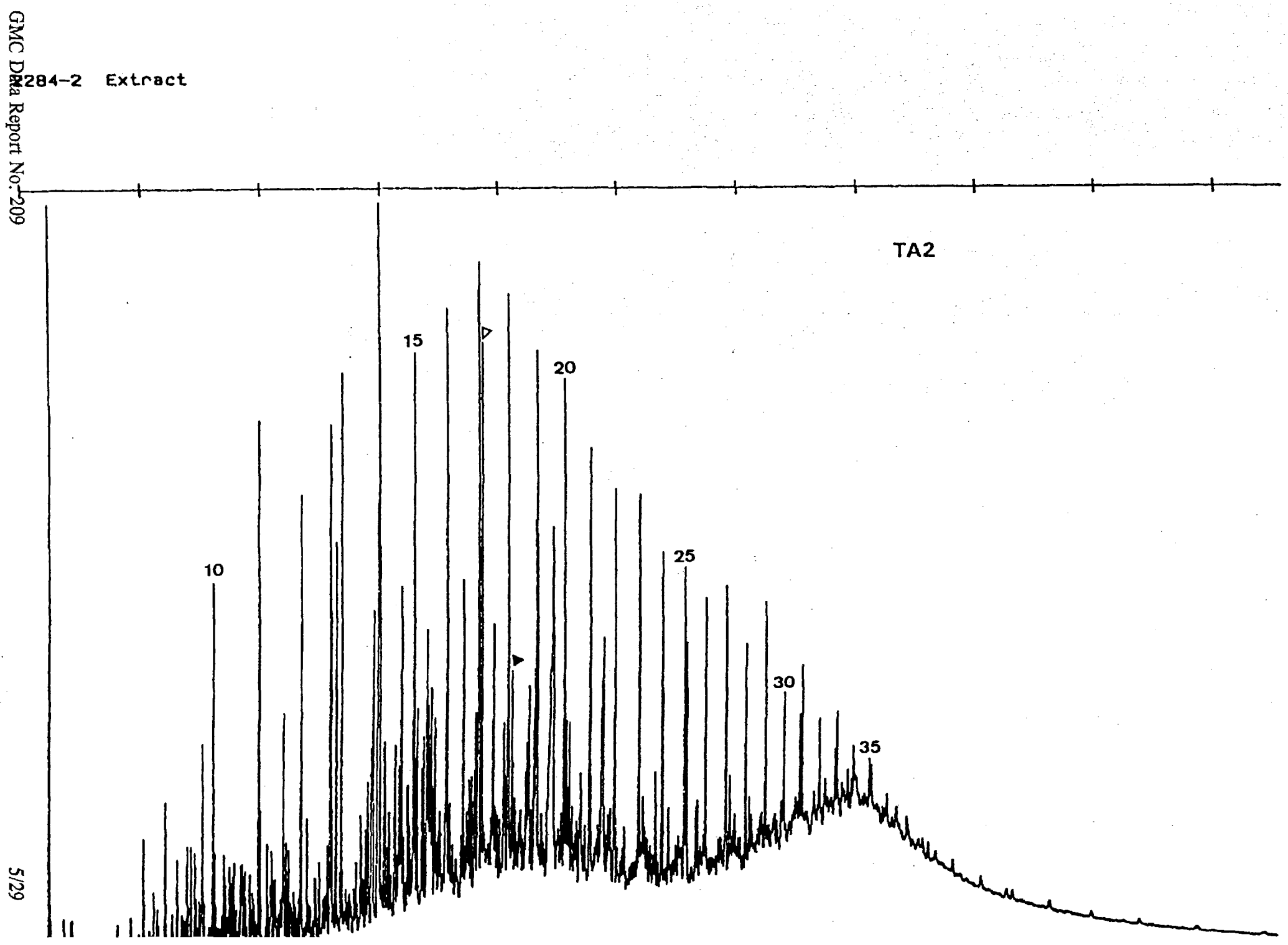




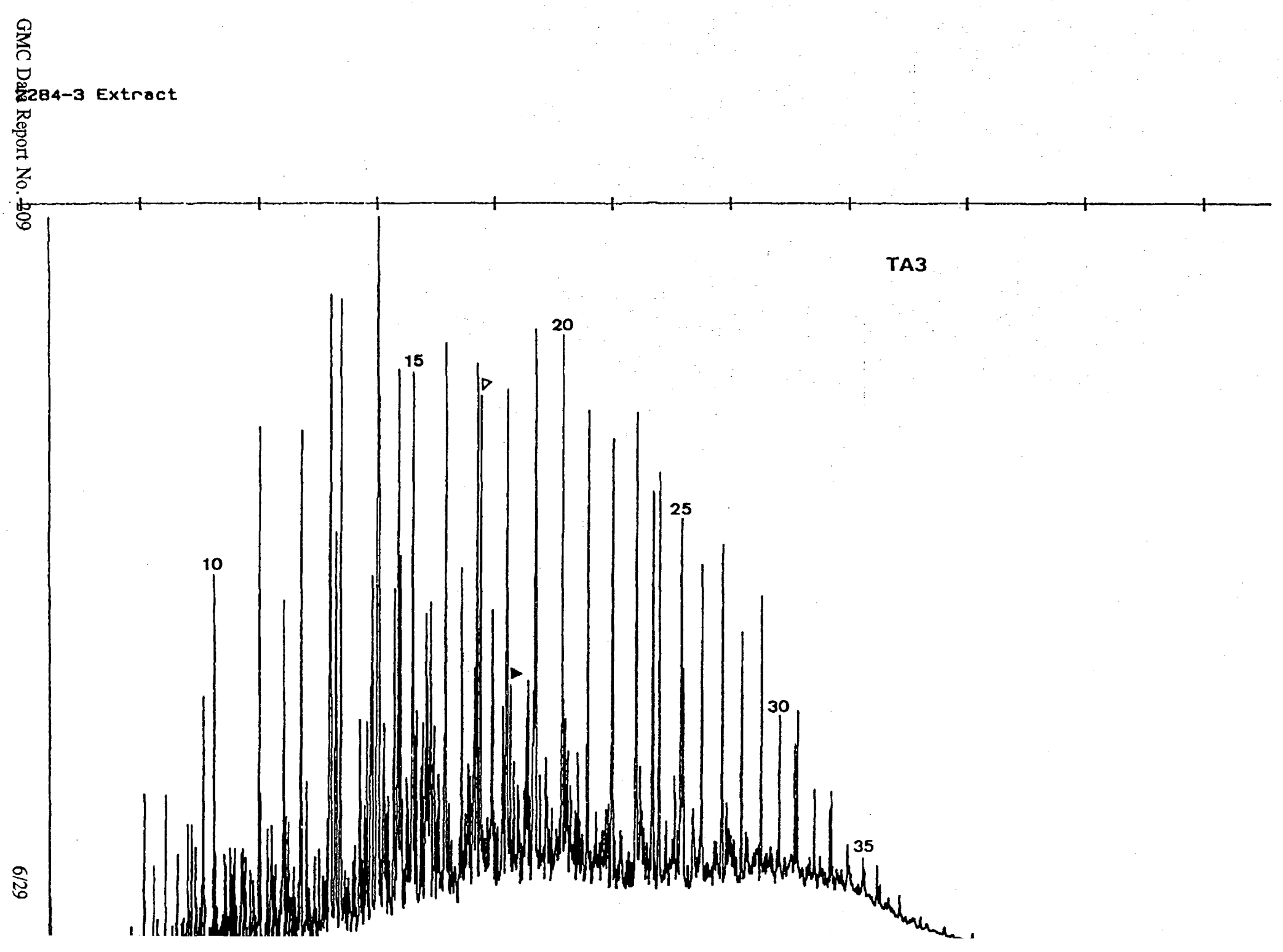


TOTAL \% COMPOSITION

Tricyclic Teroanes

C19-C29

$\mathrm{C} 30 \cdot \mathrm{C} 36$

Pentacyclic Terpanes

Hopanes

Non-Hopanes
TA1

11.53

1.06

79.97

6.38
TA2

TA3

20.55

18.50

2.82

1.24

70.63

7.75

RATIO

Tricyclic Index

57.30

110.50

97.18

C19 Tricyclic / C23 Tricyclic

0.36

0.79

0.60

C23 Tricyclic / C24 Tetracyclic

2.05

1.55

1.62

$\mathrm{Tm} / \mathrm{Ts}$

1.81

0.55

0.58

C28 Hopane / C29 Hopane

1.23

0.23

0.28

C28 Hopane / C30 Hopane

0.63

0.10

0.11

C28 Hopane /(C28 + C30 Hopanes)

0.39

0.09

0.10

C29 Hopane / C30 Hopane

0.51

0.43

0.40

C30 Moretane / C30 Hopane

0.18

0.14

0.13

C30 Oleanane / C30 Hopane

0.26

0.24

0.41

Gammacerane Index

5.56

4.58

0.00

$22 S /(22 S+22 R)$ C3i Hopane (\%)

41.67

43.61

43.98

$22 S /(22 S+22 R)$ C32 Hopane (\%)

50.64

59.66

60.97

C35 / C34 Extended Hopanes

1.42

0.64

0.61

Tricyclic / 17 aH - Hopane Ratio

0.05

0.06

0.07

C35-Homohopane Index (\%)

10.36

5.35

5.09 


\begin{tabular}{|c|c|c|c|}
\hline $\begin{array}{c}\text { TOTAL \% COMPOSITION } \\
\text { Regular steranes } \\
\text { C27 } \\
\text { C28 } \\
\text { C29 } \\
\text { Total }\end{array}$ & $\begin{array}{l}20.00 \\
21.45 \\
32.71 \\
74.16\end{array}$ & $\begin{array}{l}22.69 \\
12.68 \\
26.29 \\
61.66\end{array}$ & $\begin{array}{c}26.26 \\
8.58 \\
18.88 \\
53.72\end{array}$ \\
\hline $\begin{array}{c}\text { Rearranged (diasteranes) } \\
\text { C27 } \\
\text { C28 } \\
\text { C29 } \\
\text { Total }\end{array}$ & $\begin{array}{c}11.76 \\
6.10 \\
7.98 \\
25.84\end{array}$ & $\begin{array}{c}11.59 \\
8.31 \\
18.44 \\
38.34\end{array}$ & $\begin{array}{l}14.25 \\
12.22 \\
19.80 \\
46.28\end{array}$ \\
\hline $\begin{array}{l}\text { NORMALIZED PERCENTAGE } \\
\text { C27 aaa (2OR) } \\
\text { C28 aaa (2OR) } \\
\text { C29 aaa (2OR) }\end{array}$ & $\begin{array}{l}21.93 \\
33.01 \\
45.06\end{array}$ & $\begin{array}{l}16.78 \\
36.13 \\
47.09\end{array}$ & $\begin{array}{l}21.11 \\
28.25 \\
50.64\end{array}$ \\
\hline $\begin{array}{c}\text { NORMALIZED PERCENTAGE } \\
\text { C27 aaa }(20 R+20 S) \\
\text { C28 aaa }(20 R+20 S) \\
\text { C29 aaa }(20 R+20 S)\end{array}$ & $\begin{array}{l}25.88 \\
29.98 \\
44.15\end{array}$ & $\begin{array}{l}16.65 \\
27.22 \\
56.13\end{array}$ & $\begin{array}{l}21.34 \\
22.71 \\
55.95\end{array}$ \\
\hline \multicolumn{4}{|l|}{ RATIO } \\
\hline Diasterane Index & 43.47 & 203.47 & 266.49 \\
\hline Diasteranes / regular steranes & 0.35 & 0.62 & 0.86 \\
\hline C27 aaa (2OR) / C29 aаa (2OR) & 0.49 & 0.36 & 0.42 \\
\hline C29 aаa (20S) / C29 aaa (20R) & 0.15 & 1.06 & 1.53 \\
\hline C29 abb (2OR) / C29 aаa (2OR) & 0.17 & 1.00 & 1.36 \\
\hline $20 S /(20 S+20 R)$ C29 aàa sterane $(\%)$ & 13.16 & 51.38 & 60.54 \\
\hline$a b b /(a a a+a b b)$ C29 sterane (\%) & 31.29 & 49.17 & 51.22 \\
\hline$a b b /(a a a+a b b)$ C27 sterane (\%) & 34.12 & 82.54 & 86.63 \\
\hline Hopanes / Steranes $(\pi / S T)$ & 1.14 & 2.36 & 3.02 \\
\hline (Steranes + Diasteranes)/ C27-C30 hopanes & 1.45 & 0.77 & 0.60 \\
\hline
\end{tabular}


TAI

\begin{tabular}{|c|c|c|c|c|c|c|}
\hline Peak & 10. & Compound Name & Carbont & Ret Time & Area & Area $\%$ \\
\hline 1 & A & C19-Tricyclic Terpane & 19 & 25.382 & 157581 & 0.79 \\
\hline 2 & 8 & C20-Tricyclic Terpane & 20 & 29.869 & 210043 & 1.05 \\
\hline 3 & $\mathrm{C}$ & C21-Tricyclic Terpane & 21 & 34.568 & 205796 & 1.03 \\
\hline 4 & D & C22-Tricyclic Terpane & 22 & 39.138 & 73528 & 0.37 \\
\hline 5 & $\mathbf{E}$ & C23-Tricyclic Terpane & 23 & 44.577 & 434088 & 2.17 \\
\hline 6 & $F$ & C24-Tricyclic Terpane & 24 & 47.653 & 261568 & 1.31 \\
\hline 7 & $\mathbf{G}$ & C25-Tricyclic Terpane \#1 & 25 & 53.763 & 119509 & 0.60 \\
\hline 8 & G & C25-Tricyclic Terpane $\# 2$ & 25 & 53.943 & 128891 & 0.64 \\
\hline 9 & $\mathrm{H}$ & C24-Tetracyclic Terpane & 24 & 57.746 & 212159 & 1.06 \\
\hline 10 & 1 & C26-Tricyclic Terpane \#1 & 26 & 58.276 & 105360 & 0.53 \\
\hline 11 & 1 & C26-Tricyclic Terpane \#2 & 26 & 58.692 & 86414 & 0.43 \\
\hline 12 & $J$ & C28-Tricyclic Terpane \#1 & 28 & 68.42 & 138295 & 0.69 \\
\hline 13 & J & C28-Tricyclic Terpane $\# 2$ & 28 & 69.143 & 119594 & 0.60 \\
\hline 14 & K & C29-Tricyclic Terpane \#1 & 29 & 71.35 & 115237 & 0.58 \\
\hline 15 & $\mathbf{K}$ & C29-Tricyclic Terpane $\# 2$ & 29 & 72.203 & 152464 & 0.76 \\
\hline 16 & $\mathbf{L}$ & 18aH-Trisnorhopane (Ts) & 27 & 74.261 & 361740 & 1.81 \\
\hline 17 & $M$ & 17aH-Trisnorhopane (Tm) & 27 & 76.092 & 654502 & 3.27 \\
\hline 18 & $N$ & C30-Tricyclic Terpane \# 1 & 30 & & 0 & 0.00 \\
\hline 19 & $N$ & C30-Tricyclic Terpane $\# 2$ & 30 & & 0 & 0.00 \\
\hline 20 & 0 & 17aH,18aH,21bH-Bisnorhopane & 28 & 80.353 & 2526810 & 12.62 \\
\hline 21 & $P$ & C31-Tricyclic Terpane \#1 & 31 & & 0 & 0.00 \\
\hline 22 & $P$ & C31-Tricyclic Terpane $\# 2$ & 31 & & 0 & 0.00 \\
\hline 23 & 0 & $17 \mathrm{aH}, 21 \mathrm{bH}-$ Norhopane & 29 & 82.528 & 2059840 & 10.29 \\
\hline 24 & $R$ & $176 \mathrm{H}, 21 \mathrm{aH}-$ Normoretane & 29 & 84.634 & 1072183 & 5.36 \\
\hline 25 & $\mathbf{S}$ & 18aH-Oleanane & 30 & 85.982 & 1052829 & 5.26 \\
\hline 26 & $T$ & $17 \mathrm{aH}, 21 \mathrm{bH}-\mathrm{Hopane}$ & 30 & 86.416 & 4028500 & 20.13 \\
\hline 27 & $U$ & $17 \mathrm{bH}, 21$ aH-Moretane & 30 & 88.136 & 738874 & 3.69 \\
\hline 28 & V & C33-Tricyclic Terpane \#1 & 33 & & 0 & 0.00 \\
\hline 29 & v & C33-Tricyclic Terpane \#2 & 33 & & 0 & 0.00 \\
\hline 30 & $w$ & $17 \mathrm{aH}, 21 \mathrm{bH}-\mathrm{Homohopane}(22 \mathrm{~S})$ & 31 & 91.285 & 897572 & 4.48 \\
\hline 31 & $\mathrm{x}$ & $17 \mathrm{aH}, 21 \mathrm{bH} \cdot \mathrm{Homohopane}(22 \mathrm{R})$ & 31 & 91.91 & 1256471 & 6.28 \\
\hline 32 & Y & Gammacerane & 30 & 92.489 & 224042 & 1.12 \\
\hline 33 & $\mathbf{Z}$ & C34-Tricyclic Terpane \#1 & 34 & & 0 & 0.00 \\
\hline 34 & $\mathbf{Z}$ & C34-Tricyclic Terpane $\# 2$ & 34 & & 0 & 0.00 \\
\hline 35 & a & $17 \mathrm{aH}, 21 \mathrm{bH}-$ Bishomohopane (22S) & 32 & 95.66 & 502984 & 2.51 \\
\hline 36 & b & $17 \mathrm{aH}, 21 \mathrm{bH}-$ Bishomohopane (22R) & 32 & 96.664 & 490253 & 2.45 \\
\hline 37 & c & C35-Tricyclic Terpane \#1 & 35 & 98.811 & 118957 & 0.59 \\
\hline 38 & c & C35-Tricyclic Terpane $\# 2$ & 35 & 100.517 & 93487 & 0.47 \\
\hline 39 & d & $17 \mathrm{aH}, 21 \mathrm{bH}-$ Trishomohopane (22S) & 33 & 101.529 & 285627 & 1.43 \\
\hline 40 & e & 17aH,21bH-Trishomohopane (22R) & 33 & 103.161 & 326564 & 1.63 \\
\hline 41 & 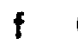 & C36-Tricyclic Terpane \#1 & 36 & & 0 & 0.00 \\
\hline 42 & f & C36-Tricyclic Terpane $\# 2$ & 36 & & 0 & 0.00 \\
\hline 43 & g & 17aH,21bH-Tetrahomohopane (22S) & 34 & 108.747 & 165246 & 0.83 \\
\hline 44 & h & 17aH,21bH-Tetrahomohopane (22R) & 34 & 111.128 & 166664 & 0.83 \\
\hline 45 & $\mathbf{i}$ & 17aH,21bH-Pentahomohopane (22S) & 35 & 117.267 & 194734 & 0.97 \\
\hline 46 & j & $17 \mathrm{aH}, 21 \mathrm{bH}$-Pentahomohopane (22R) & 35 & 120.756 & 278209 & 1.39 \\
\hline
\end{tabular}




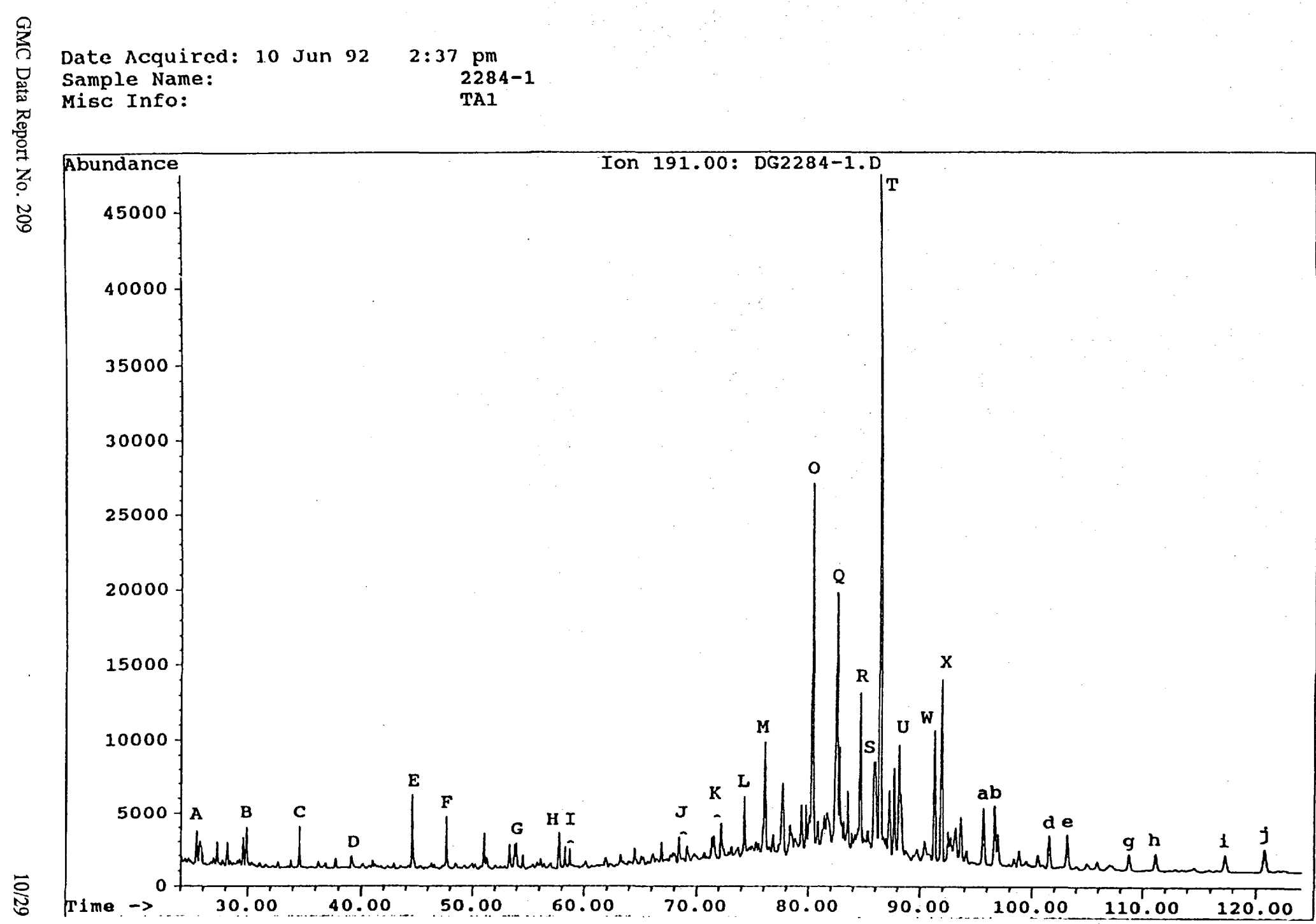




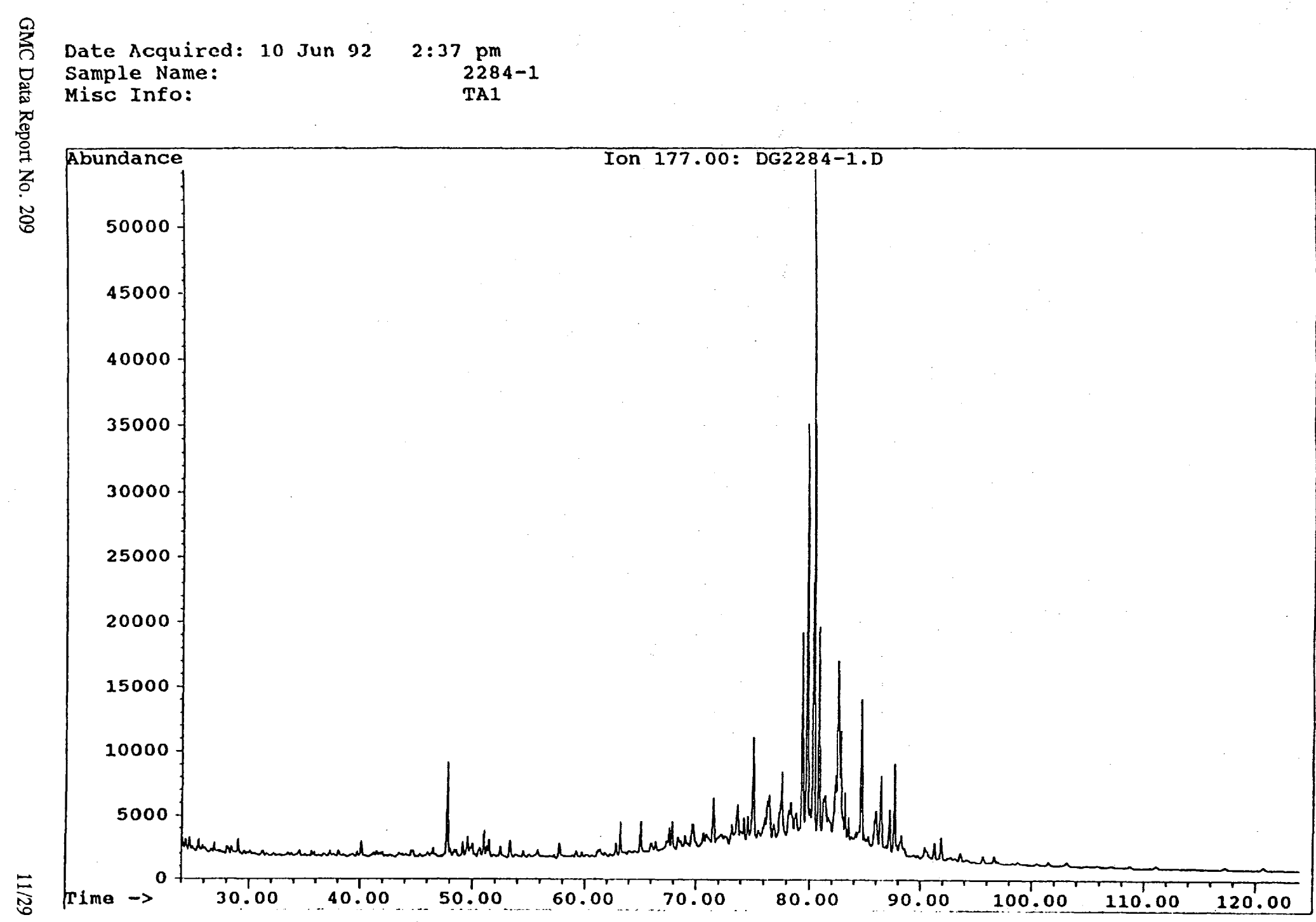


TA1

\begin{tabular}{lll} 
Peak ID. & \multicolumn{1}{c}{ Sterane Name } \\
1 & A & C27 ba diasterane (2OS) \\
2 & B & C27 ba diasterane (2OR) \\
3 & C & C27 ab diasterane (2OS) \\
4 & D & C27 ab diasterane (2OR) \\
5 & E & C28 ba diasterane (2OS) \\
6 & F & C28 ba diasterane (2OR) \\
7 & G & C27 aaa (2OS) + C28 ab dia-(20S) \\
8 & H & C27 abb (2OR) + C29 ba dia-(20S) \\
9 & I & C27 abb (20S) + C28 ab dia-(2OR) \\
10 & J & C27 aaa sterane (2OR) \\
11 & K & C29 ba diasterane (2OR) \\
12 & L & C29 ab diasterane (2OS) \\
13 & M & C28 aaa sterane (2OS) \\
14 & N & C28 abb sterane (2OR) \\
15 & O & C28 abb sterane (2OS) \\
16 & P & C28 aaa sterane (2OR) \\
17 & Q & C29 aaa sterane (20S) \\
18 & R & C29 abb sterane (2OR) \\
19 & S & C29 abb sterane (2OS) \\
20 & T & C29 aaa sterane (2OR)
\end{tabular}

\begin{tabular}{rrc} 
Ret Time & \multicolumn{1}{c}{ Area } & Area\% \\
63.215 & 583986 & 3.09 \\
65.049 & 497936 & 2.64 \\
66.429 & 282349 & 1.49 \\
67.474 & 857630 & 4.54 \\
67.874 & 876491 & 4.64 \\
69.81 & 275607 & 1.46 \\
71.245 & 694214 & 3.67 \\
71.559 & 981532 & 5.20 \\
71.962 & 307653 & 1.63 \\
73.171 & 1794783 & 9.50 \\
73.689 & 1042164 & 5.52 \\
74.613 & 465416 & 2.46 \\
75.827 & 182525 & 0.97 \\
76.475 & 712321 & 3.77 \\
76.849 & 457035 & 2.42 \\
78.308 & 2701078 & 14.30 \\
79.69 & 558700 & 2.96 \\
80.433 & 622527 & 3.29 \\
80.654 & 1311520 & 6.94 \\
82.497 & 3687630 & 19.52
\end{tabular}




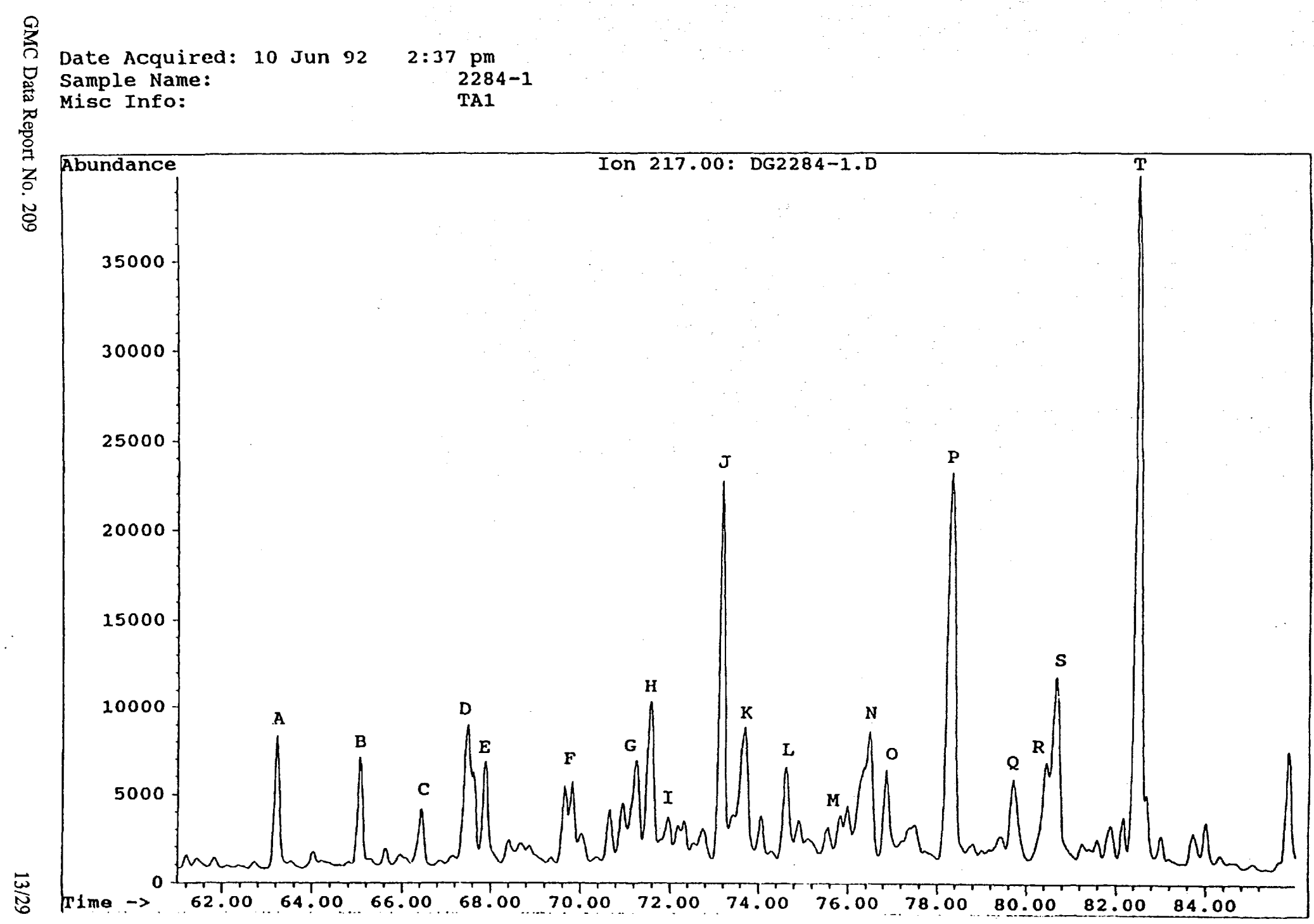




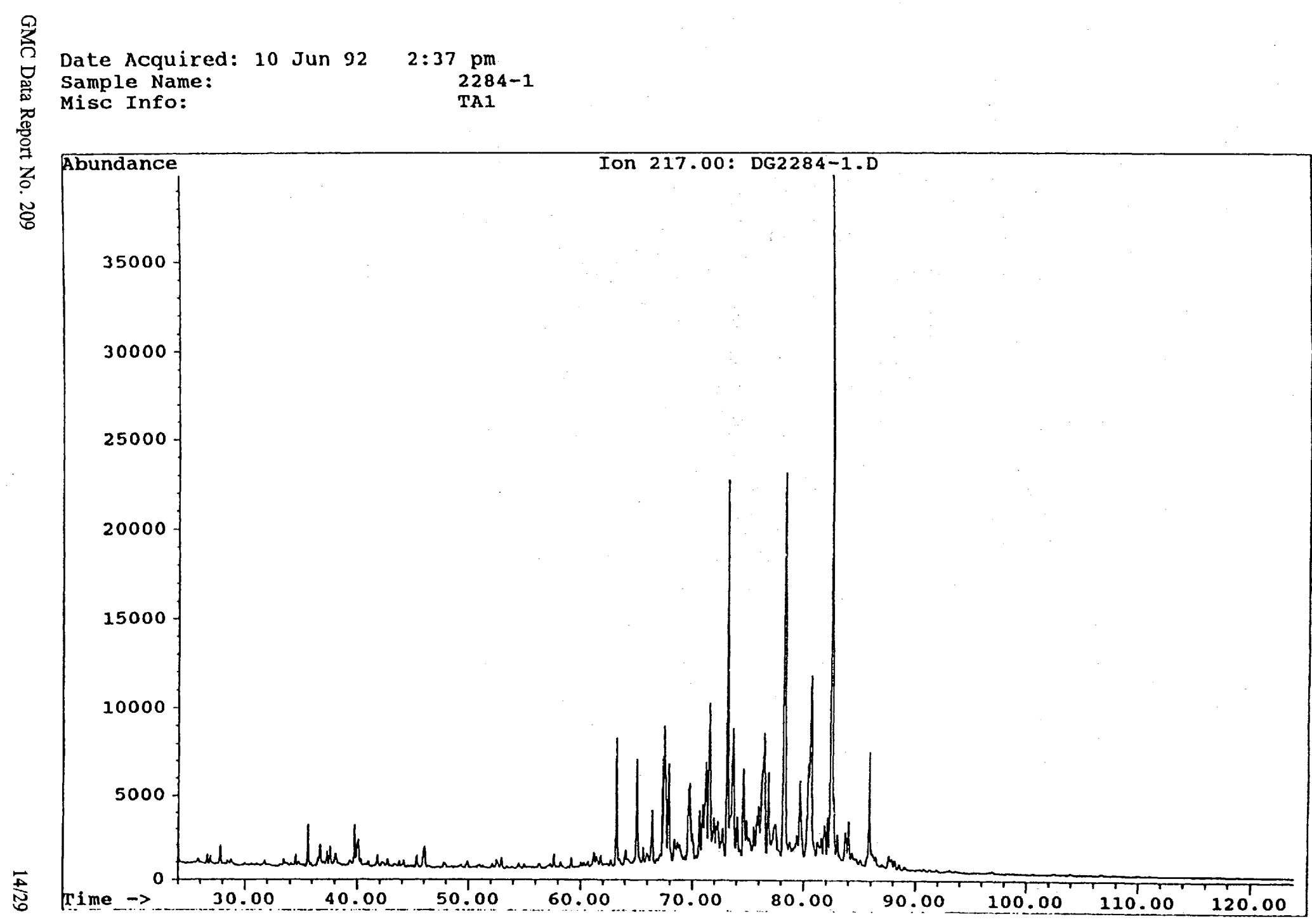




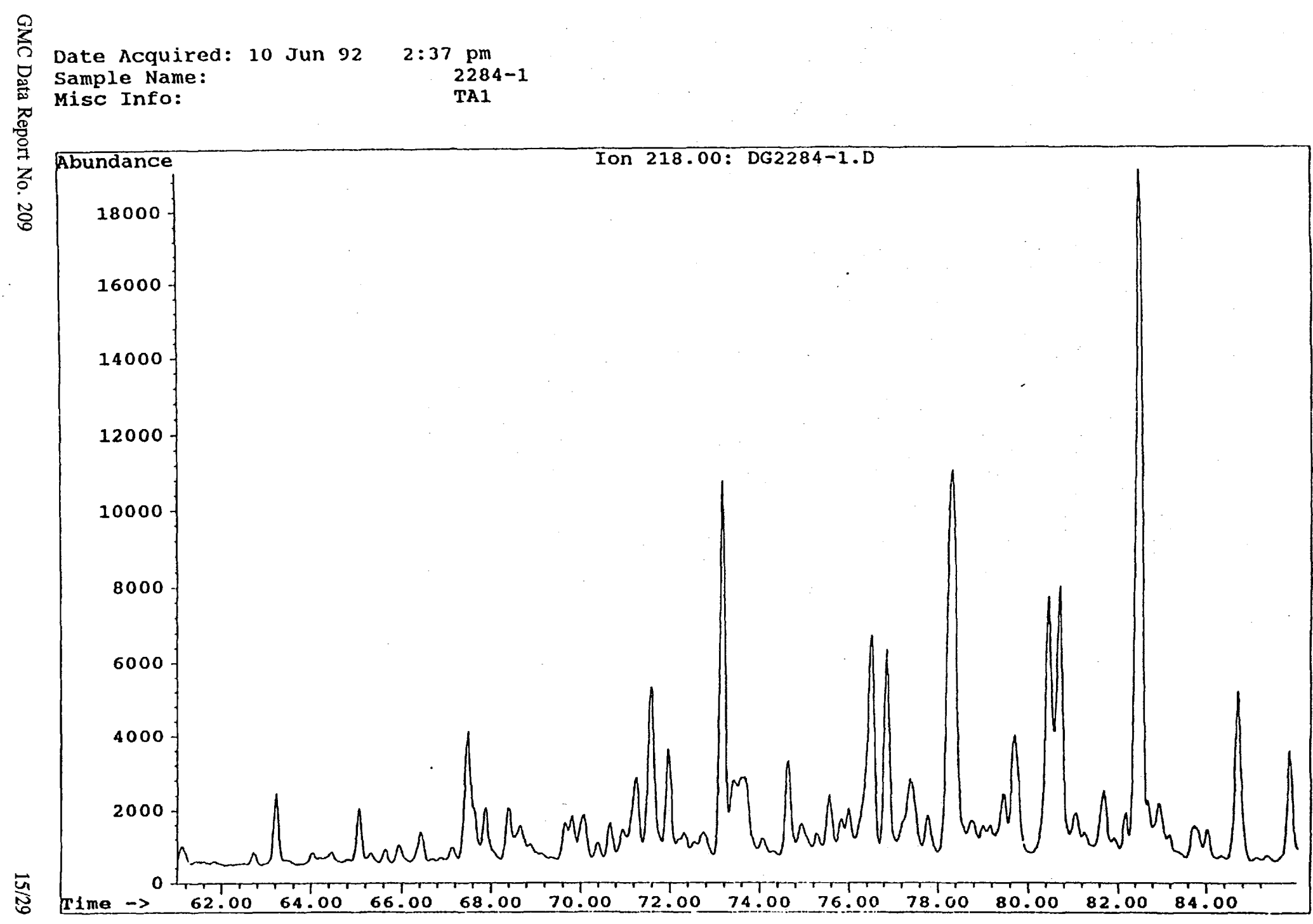


TA2

\begin{tabular}{|c|c|c|c|c|c|c|}
\hline \multicolumn{2}{|c|}{ Peak ID. } & Compound Name & Carbon: & Ret Time & Area & Area\% \\
\hline 1 & A & C19-Tricyclic Terpane & 19 & 25.713 & 450416 & 2.47 \\
\hline 2 & $\mathbf{B}$ & C20-Tricyclic Terpane & 20 & 30.141 & 690018 & 3.79 \\
\hline 3 & C & C21-Trisyclic Terpane & 21 & 34.809 & 482421 & 2.65 \\
\hline 4 & D & C22-Tricyclic Terpane & 22 & 39.35 & 109221 & 0.60 \\
\hline 5 & $\mathbf{E}$ & C23-Tricyclic Terpane & 23 & 44.776 & 573636 & 3.15 \\
\hline 6 & $\mathbf{F}$ & C24-Tricyclic Terpane & 24 & 47.854 & 364893 & 2.00 \\
\hline 7 & G & C25-Tricyclic Terpane \#1 & 25 & 53.948 & 109975 & 0.60 \\
\hline 8 & G & C25-Tricyclic Terpane $\# 2$ & 25 & 54.137 & 139649 & 0.77 \\
\hline 9 & $\mathrm{H}$ & C24-Tetracyclic Terpane & 24 & 57.95 & 368914 & 2.02 \\
\hline 10 & 1 & C26-Tricyclic Terpane \# 1 & 26 & 58.475 & 130723 & 0.72 \\
\hline 11 & 1 & C26-Tricyclic Terpane \#2 & 26 & 58.893 & 104386 & 0.57 \\
\hline 12 & $J$ & C28-Tricyclic Terpane \#1 & 28 & 68.628 & 196576 & 1.08 \\
\hline 13 & $\mathbf{J}$ & C28-Tricyclic Terpane \#2 & 28 & 69.348 & 136419 & 0.75 \\
\hline 14 & $k$ & C29-Tricyclic Terpane \#1 & 29 & 71.581 & 178691 & 0.98 \\
\hline 15 & $K$ & C29-Tricyclic Terpane $\# 2$ & 29 & 72.431 & 78486 & 0.43 \\
\hline 16 & $L$ & 18aH-Trisnorhopane (Ts) & 27 & 74.477 & 1160619 & 6.37 \\
\hline 17 & $M$ & 17aH-Trisnorhopane (Tm) & 27 & 76.3 & 633989 & 3.48 \\
\hline 18 & $N$ & C30-Tricyclic Terpane \#1 & 30 & & 0 & 0.00 \\
\hline 19 & $N$ & C30-Tricyclic Terpane \#2 & 30 & & 0 & 0.00 \\
\hline 20 & 0 & 17aH,18aH,21bH-Bisnorhopane & 28 & 80.523 & 334362 & 1.83 \\
\hline 21 & $P$ & C31-Tricyclic Terpane \#1 & 31 & & 0 & 0.00 \\
\hline 22 & $\mathbf{P}$ & C31-Tricyclic Terpane \#2 & 31 & & 0 & 0.00 \\
\hline 23 & 0 & 17aH,21bH-Norhopane & 29 & 82.718 & 1456583 & 7.99 \\
\hline 24 & $R$ & $17 \mathrm{bH}, 21 \mathrm{aH}-\mathrm{Normoretane}$ & 29 & 84.809 & 278502 & 1.53 \\
\hline 25 & $\mathbf{s}$ & $18 a \mathrm{H}$-Oleanane & 30 & 86.04 & 798199 & 4.38 \\
\hline 26 & $T$ & 17aH,216H-Hopane & 30 & 86.605 & 3389670 & 18.60 \\
\hline 27 & $U$ & 17bH,21 aH-Moretane & 30 & 88.345 & 471018 & 2.58 \\
\hline 28 & V & C33-Tricyclic Terpane $\# 1$ & 33 & & 0 & 0.00 \\
\hline 29 & V & C33-Tricyclic Terpane \#2 & 33 & & 0 & 0.00 \\
\hline 30 & W & 17aH,21bH-Homohopane (22S) & 31 & 91.532 & 1002656 & 5.50 \\
\hline 31 & $x$ & $17 \mathrm{aH}, 21 \mathrm{bH}-\mathrm{H} o$ mohopane $(22 \mathrm{R})$ & 31 & 92.156 & 1296255 & 7.11 \\
\hline 32 & $Y$ & Gammacerane & 30 & 92.736 & 155115 & 0.85 \\
\hline 33 & $\mathbf{z}$ & C34-Tricyclic Terpane \#1 & 34 & & 0 & 0.00 \\
\hline 34 & $\bar{z}$ & C34-Tricyclic Terpane $\# 2$ & 34 & & 0 & 0.00 \\
\hline 35 & $\mathbf{a}$ & 17aH,21bH-Bishomohopane (22S) & 32 & 95.936 & 688802 & 3.78 \\
\hline 36 & b & $17 \mathrm{aH}, 21 \mathrm{bH}-$ Bishomohopane (22R) & 32 & 96.931 & 465743 & 2.56 \\
\hline 37 & c & C35-Tricyclic Terpane \#1 & 35 & 99.113 & 141711 & 0.78 \\
\hline 38 & c & C35-Tricyclic Terpane \#2 & 35 & 100.806 & 83133 & 0.46 \\
\hline 39 & $d$ & 17aH,21bH-Trishomohopane (22S) & 33 & 101.827 & 461524 & 2.53 \\
\hline 40 & e & $17 \mathrm{aH}, 21 \mathrm{bH}-\mathrm{Trishomohopane}(22 \mathrm{R})$ & 33 & 103.462 & 330739 & 1.81 \\
\hline 41 & $f$ & C36-Tricyclic Terpane \#1 & 36 & 105.268 & 120885 & 0.66 \\
\hline 42 & $f$ & C36-Tricyclic Terpane $\# 2$ & 36 & 107.379 & 168161 & 0.92 \\
\hline 43 & 0 & 17aH.21bH-Tetrahomohopane (22S) & 34 & 109.07 & 237974 & 1.31 \\
\hline 44 & $\mathrm{~h}$ & $17 \mathrm{aH}, 21 \mathrm{bH}-\mathrm{Tetrahomohopane}(22 \mathrm{R})$ & 34 & 111.464 & 172100 & 0.94 \\
\hline 45 & i & 17aH,21bH-Pentahomohopane (22S) & 35 & 117.644 & 155752 & 0.85 \\
\hline 46 & j & $17 \mathrm{aH}, 21 \mathrm{bH}-$ Pentahomohopane (22R) & 35 & 121.145 & 107536 & 0.59 \\
\hline
\end{tabular}


Abundance

35000

35000

30000

3000

売 


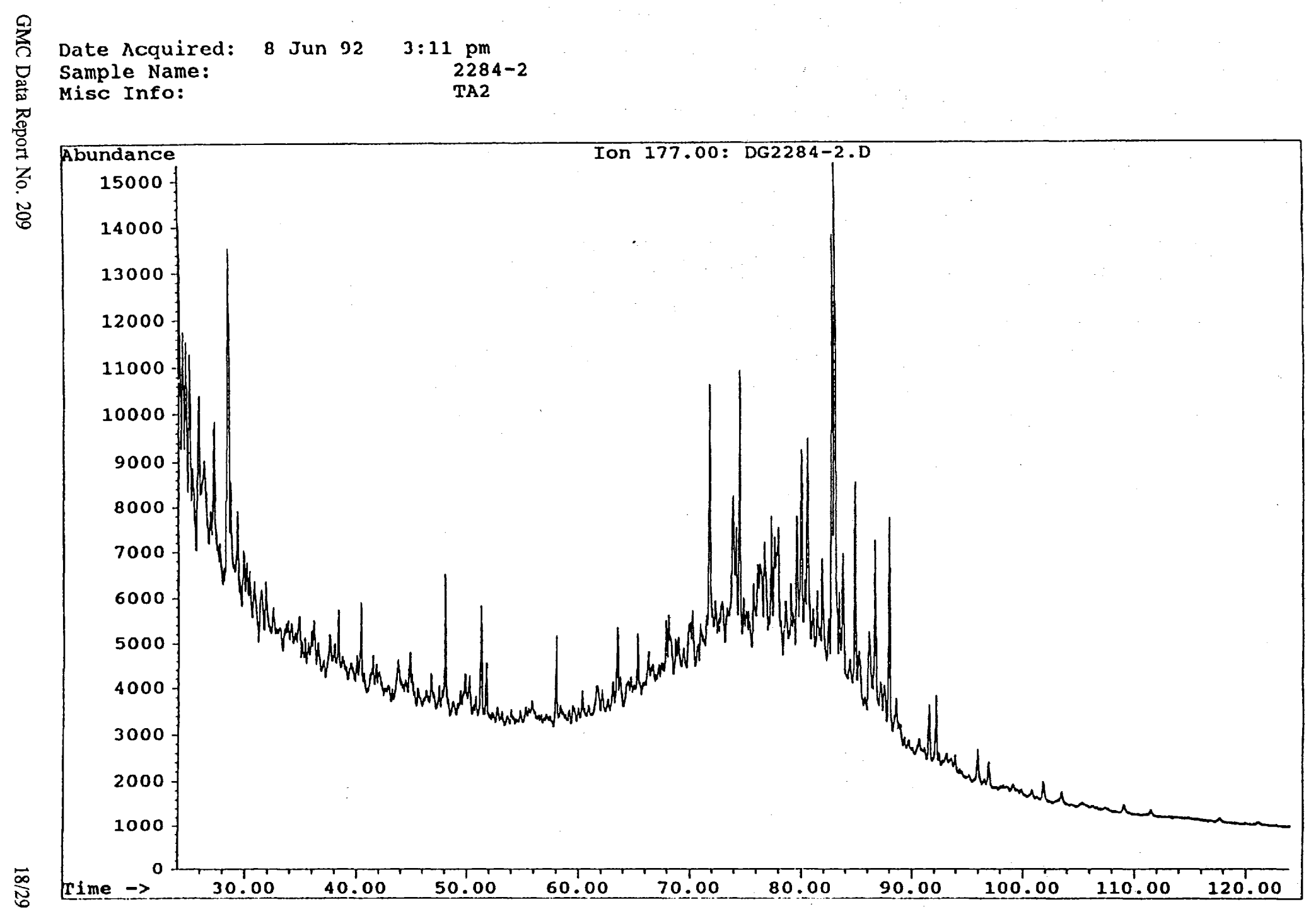


TA2

$\begin{array}{lll}\text { Peak ID. } & \text { Sterane Name } \\ 1 & \text { A } & \text { C27 ba diasterane (2OS) } \\ 2 & \text { B } & \text { C27 ba diasterane (2OR) } \\ 3 & \text { C } & \text { C27 ab diasterane (2OS) } \\ 4 & \text { D } & \text { C27 ab diasterane (2OR) } \\ 5 & \text { E } & \text { C28 ba diasterane (2OS) } \\ 6 & \text { F } & \text { C28 ba diasterane (2OR) } \\ 7 & \text { G } & \text { C27 aaa (2OS) + C28 ab dia-(2OS) } \\ 8 & \text { H } & \text { C27 abb (2OR) + C29 ba dia-(2OS) } \\ 9 & \text { I } & \text { C27 abb (2OS) + C28 ab dia-(2OR) } \\ 10 & \text { J } & \text { C27 aaa sterane (2OR) } \\ 11 & \text { K } & \text { C29 ba diasterane (2OR) } \\ 12 & \text { L } & \text { C29 ab diasterane (2OS) } \\ 13 & \text { M } & \text { C28 aaa sterane (2OS) } \\ 14 & \text { N } & \text { C28 abb sterane (2OR) } \\ 15 & \text { O } & \text { C28 abb sterane (2OS) } \\ 16 & \text { P } & \text { C28 aaa sterane (2OR) } \\ 17 & \text { Q } & \text { C29 aaa sterane (20S) } \\ 18 & \text { R } & \text { C29 abb sterane (2OR) } \\ 19 & \text { S } & \text { C29 abb sterane (2OS) } \\ 20 & \text { T } & \text { C29 aaa sterane (2OR) }\end{array}$

\begin{tabular}{rrc} 
Ret Time & \multicolumn{1}{c}{ Area } & Area\% \\
63.414 & 468159 & 5.38 \\
65.252 & 233304 & 2.68 \\
66.631 & 176497 & 2.03 \\
67.596 & 129828 & 1.49 \\
68.086 & 520247 & 5.98 \\
70.017 & 202938 & 2.33 \\
71.394 & 143298 & 1.65 \\
71.774 & 1472923 & 16.93 \\
72.173 & 156359 & 1.80 \\
73.37 & 201449 & 2.32 \\
73.9 & 1206718 & 13.87 \\
74.815 & 397494 & 4.57 \\
76.043 & 130076 & 1.50 \\
76.691 & 229871 & 2.64 \\
77.061 & 309126 & 3.55 \\
78.483 & 433656 & 4.99 \\
79.854 & 597229 & 6.87 \\
80.652 & 562567 & 6.47 \\
80.883 & 561844 & 6.46 \\
82.648 & 565204 & 6.50
\end{tabular}




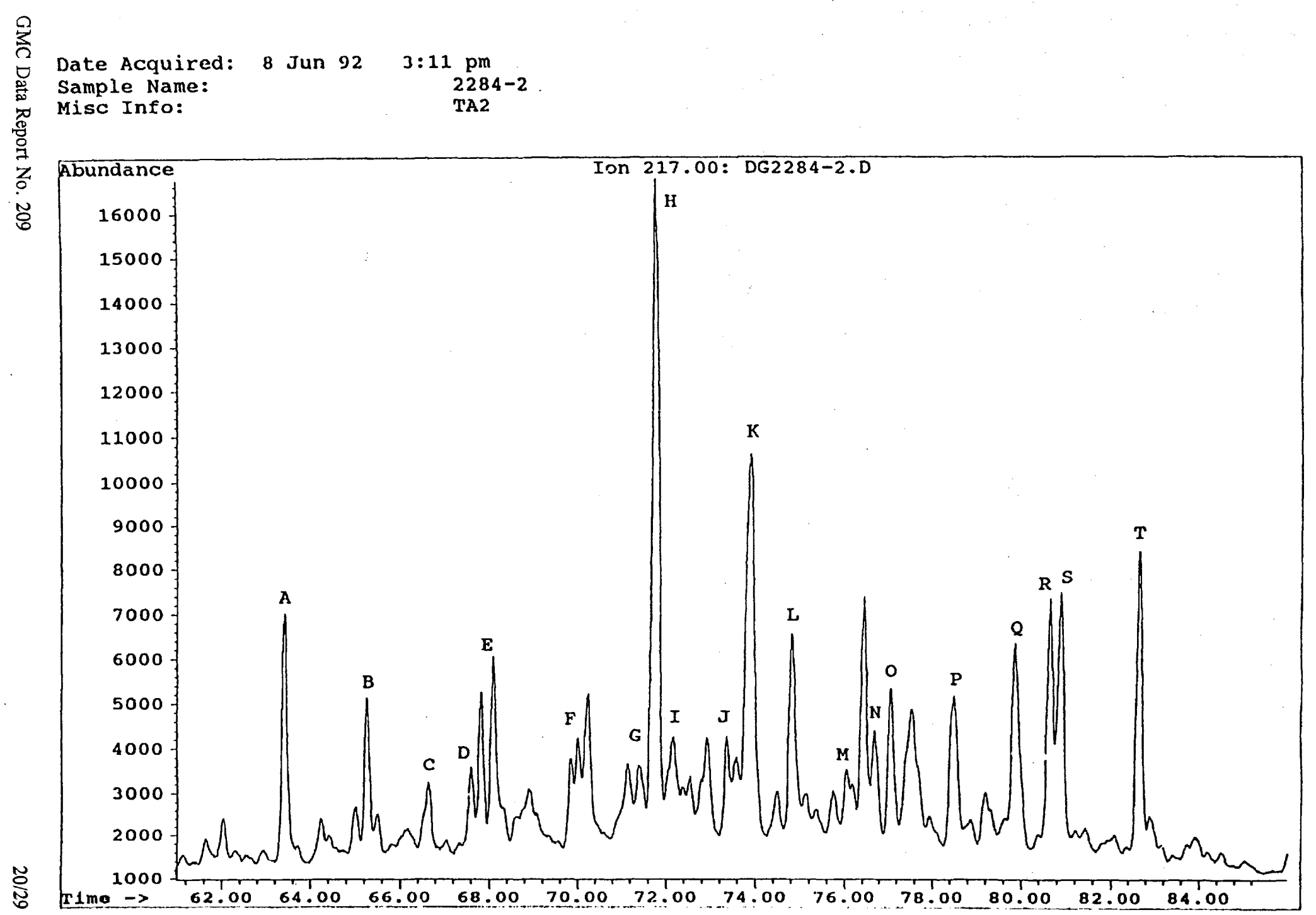




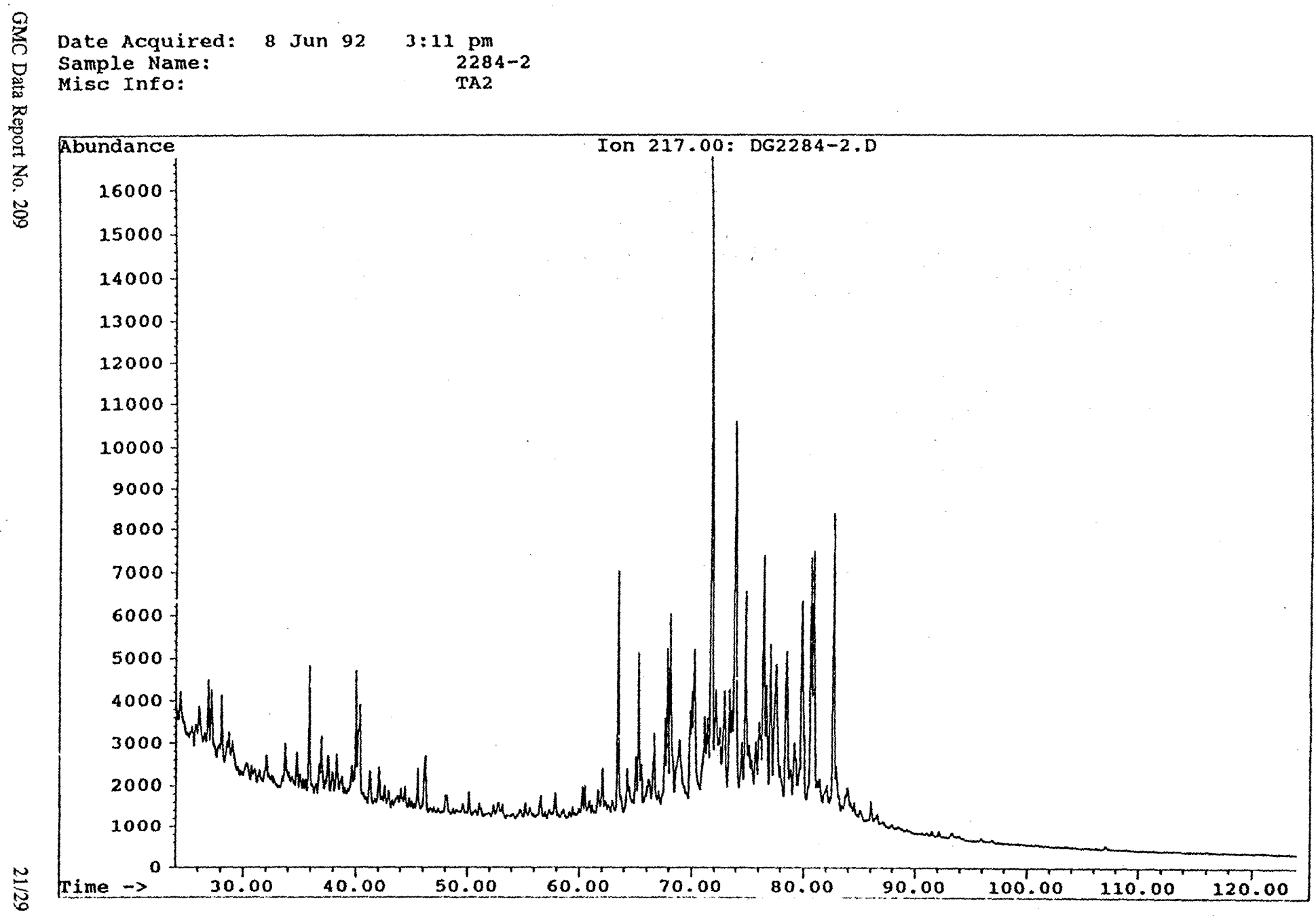


Date Acquired: 8 Jun $92 \quad 3: 11 \mathrm{pm}$ Sample Name: 2284-2

Misc Info:

TA2

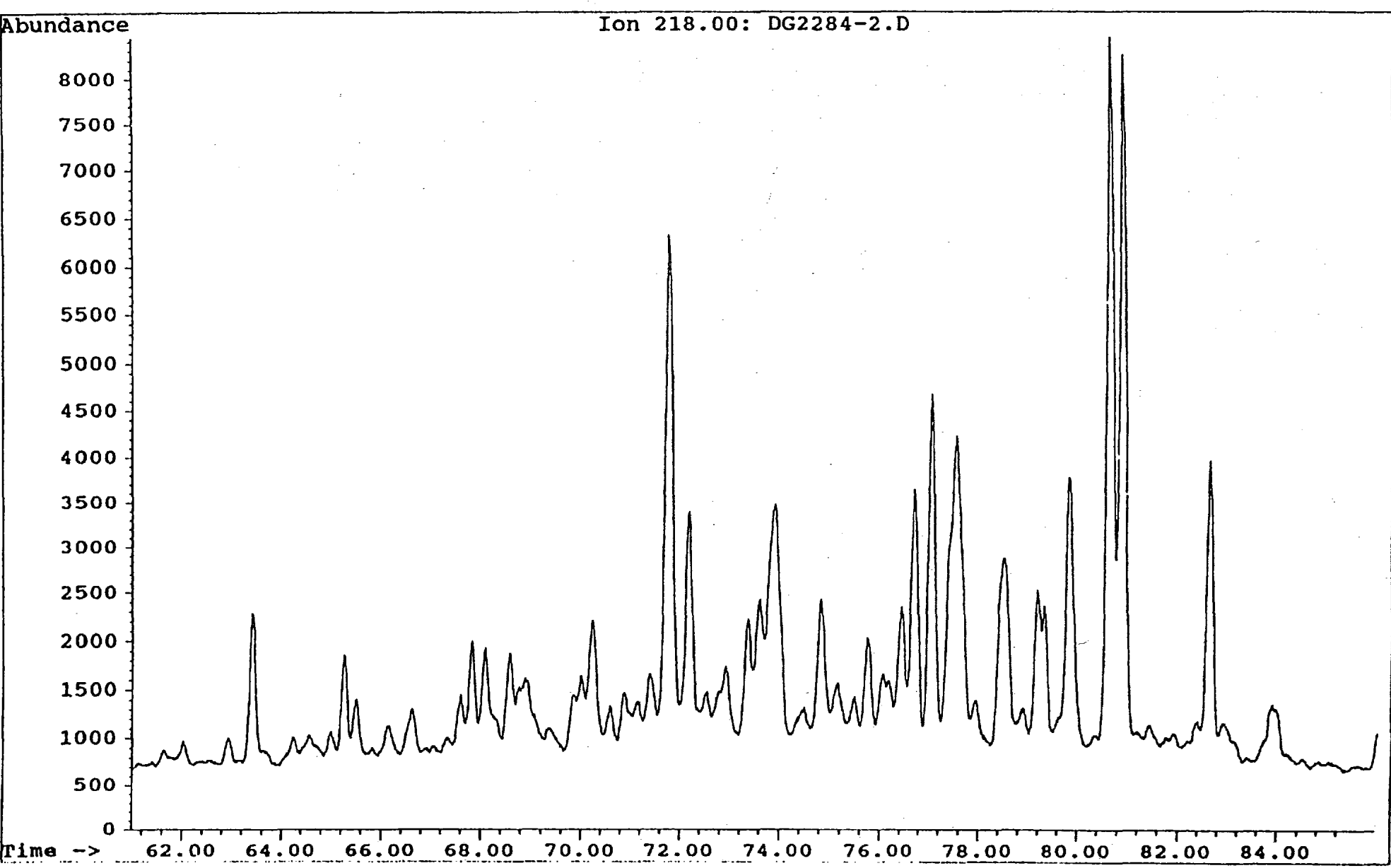


TA3

\begin{tabular}{|c|c|c|c|c|c|c|}
\hline \multicolumn{2}{|c|}{ Peak ID. } & Compound Name & Carbon \# & Ret Time & Area & Area\% \\
\hline 1 & $A$ & C19-Tricyclic Terpane & 19 & 25.747 & 408369 & 1.84 \\
\hline 2 & $\mathbf{8}$ & C20-Tricyclic Terpane & 20 & 29.862 & 580074 & 2.62 \\
\hline 3 & C & C21-Tricyclic Terpane & 21 & 34.828 & 478422 & 2.16 \\
\hline 4 & $D$ & C22-Tricyclic Terpane & 22 & 39.382 & 138367 & 0.62 \\
\hline 5 & $E$ & C23-Tricyclic Terpane & 23 & 44.819 & 675175 & 3.04 \\
\hline 6 & $\mathbf{F}$ & C24-Tricyclic Terpane & 24 & 47.908 & 471357 & 2.13 \\
\hline 7 & G & C25-Tricyclic Terpane \#1 & 25 & 53.995 & 142353 & 0.64 \\
\hline 8 & G & C25-Tricyclic Terpane $\# 2$ & 25 & 54.183 & 134842 & 0.61 \\
\hline 9 & $H$ & C24-Tetracyclic Terpane & 24 & 57.998 & 417799 & 1.88 \\
\hline 10 & 1 & C26-Tricyclic Terpane \#1 & 26 & 58.522 & 163917 & 0.74 \\
\hline 11 & 1 & C26-Tricyclic Terpane $\# 2$ & 26 & 58.933 & 131290 & 0.59 \\
\hline 12 & 小 & C28-Tricyclic Terpane \#1 & 28 & 68.683 & 224335 & 1.01 \\
\hline 13 & J & C28-Tricyclic Terpane $\# 2$ & 28 & 69.382 & 141701 & 0.64 \\
\hline 14 & K & C29-Tricyclic Terpane \#1 & 29 & 71.635 & 249415 & 1.12 \\
\hline 15 & $K$ & C29-Tricyclic Terpane $\# 2$ & 29 & 72.471 & 163284 & 0.74 \\
\hline 16 & $\mathrm{~L}$ & 18aH-Trisnorhopane (Ts) & 27 & 74.525 & 1463862 & 6.60 \\
\hline 17 & $M$ & 17aH-Trisnorhopane (Tm) & 27 & 76.159 & 848087 & 3.82 \\
\hline 18 & $N$ & C30-Tricyclic Terpane \#1 & 30 & & 0 & 0.00 \\
\hline 19 & $N$ & C30-Tricyclic Terpane $\# 2$ & 30 & & 0 & 0.00 \\
\hline 20 & 0 & $17 \mathrm{aH}, 18 \mathrm{aH}, 21 \mathrm{bH}-\mathrm{Bisnorhopane}$ & 28 & 80.558 & 475293 & 2.14 \\
\hline 21 & $P$ & C31-Tricyclic Terpane \#1 & 31 & & 0 & 0.00 \\
\hline 22 & $\mathbf{P}$ & C31-Tricyclic Terpane $\# 2$ & 31 & & 0 & 0.00 \\
\hline 23 & 0 & 17aH,21bH-Norhopane & 29 & 82.74 & 1686306 & 7.60 \\
\hline 24 & $R$ & $17 \mathrm{bH}, 21 \mathrm{aH}-$ Normoretane & 29 & 84.833 & 329620 & 1.49 \\
\hline 25 & $\mathbf{S}$ & 18aH-Oleanane & 30 & 86.064 & 1718646 & 7.75 \\
\hline 26 & $T$ & 17aH,21bH-Hopane & 30 & 86.627 & 4222131 & 19.04 \\
\hline 27 & $U$ & $17 \mathrm{bH}, 21$ aH-Moretane & 30 & 88.36 & 534144 & 2.41 \\
\hline 28 & V & C33-Tricyclic Terpane $\# 1$ & 33 & & 0 & 0.00 \\
\hline 29 & V & C33-Tricyelic Terpane $\# 2$ & 33 & & 0 & 0.00 \\
\hline 30 & $w$ & $17 \mathrm{aH}, 21 \mathrm{bH}-\mathrm{H}$ omohopane (22S) & 31 & 91.546 & 1260832 & 5.69 \\
\hline 31 & $x$ & 17aH,21bH-Homohopane (22R) & 31 & 92.17 & 1606177 & 7.24 \\
\hline 32 & Y & Gammacerane & 30 & & 0 & 0.00 \\
\hline 33 & $\mathbf{z}$ & C34-Tricyclic Terpane $\# 1$ & 34 & & 0 & 0.00 \\
\hline 34 & $\mathbf{z}$ & C34-Tricyclic Terpane $\# 2$ & 34 & & 0 & 0.00 \\
\hline 35 & $\mathbf{a}$ & 17aH,21bH-Bisnomohopane (22S) & 32 & 95.944 & 877189 & 3.96 \\
\hline 36 & b & 17aH,21bH-Bishomohopane (22R) & 32 & 96.937 & 561444 & 2.53 \\
\hline 37 & c & C35-Tricyclic Terpane $\# 1$ & 35 & 99.118 & 166264 & 0.75 \\
\hline 38 & c & C35-Tricyclic Terpane \#2 & 35 & 100.812 & 108336 & 0.49 \\
\hline 39 & $d$ & 17aH, $21 \mathrm{bH}$-Trishomohopane (22S) & 33 & 101.83 & 587809 & 2.65 \\
\hline 40 & e & 17aH,21bH-Trishomohopane (22R) & 33 & 103.469 & 391965 & 1.77 \\
\hline 41 & $f$ & C36-Tricyclic Terpane \#1 & 36 & & 0 & 0.00 \\
\hline 42 & $f$ & C36-Tricyclic Terpane \#2 & 36 & & 0 & 0.00 \\
\hline 43 & g & 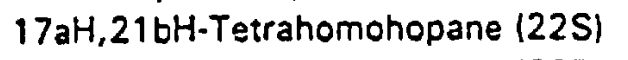 & 34 & 109.07 & 314574 & 1.42 \\
\hline 44 & $h$ & 17aH,21 bH-Tetrahomohopane (22R) & 34 & 111.45 & 191364 & 0.86 \\
\hline 45 & $\mathbf{i}$ & $17 \mathrm{aH}, 21 \mathrm{bH}-\mathrm{Pentahomohopane}(22 \mathrm{~S})$ & 35 & 117.608 & 186022 & 0.84 \\
\hline 46 & j & $17 \mathrm{aH}, 21 \mathrm{bH}-\mathrm{Pentahomohopane}(22 \mathrm{R})$ & 35 & 121.11 & 124469 & 0.56 \\
\hline
\end{tabular}




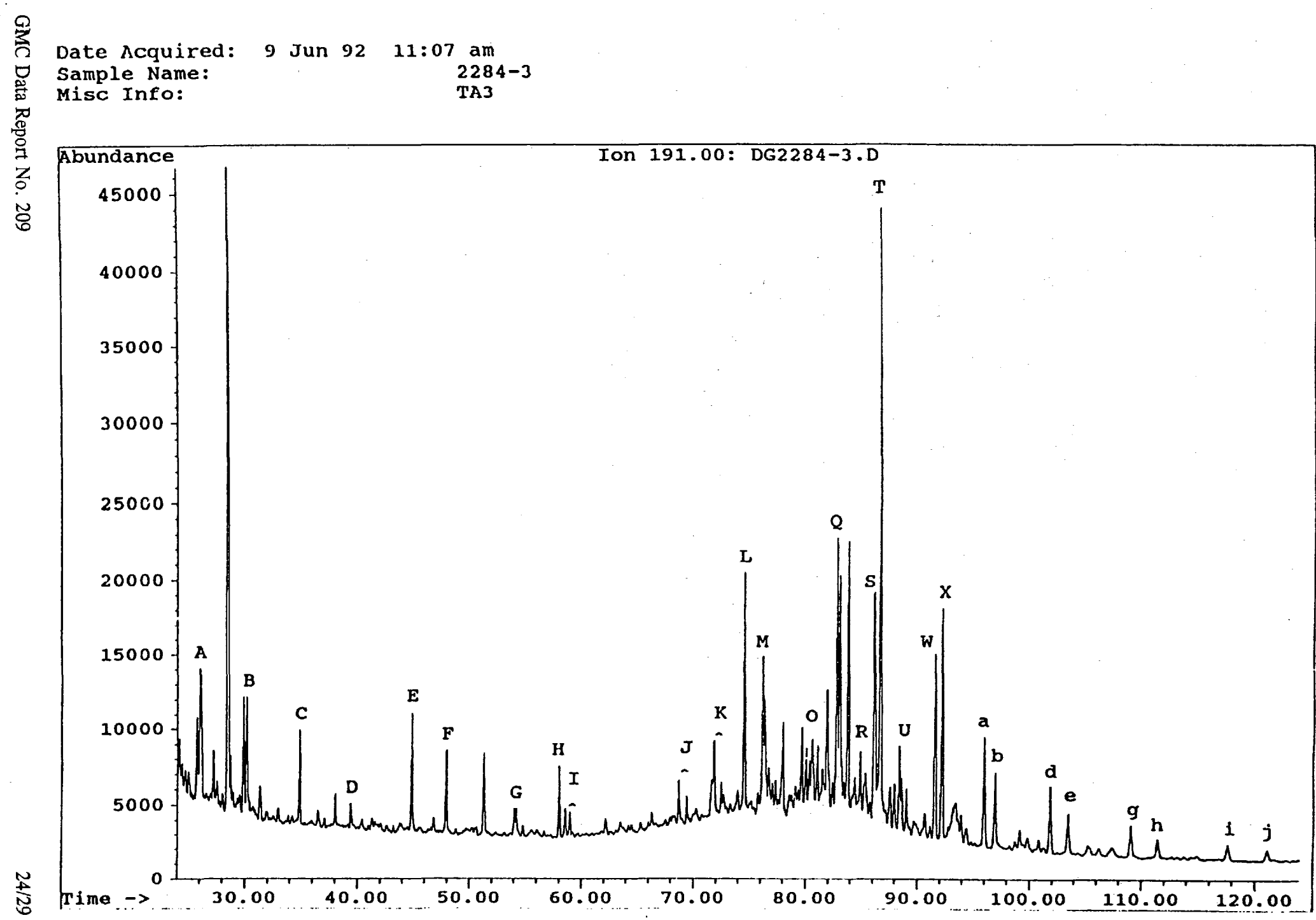


Date Acquired: 9 Jun 92 11:07 am Sample Name: 2284-3

Misc Info: TA3

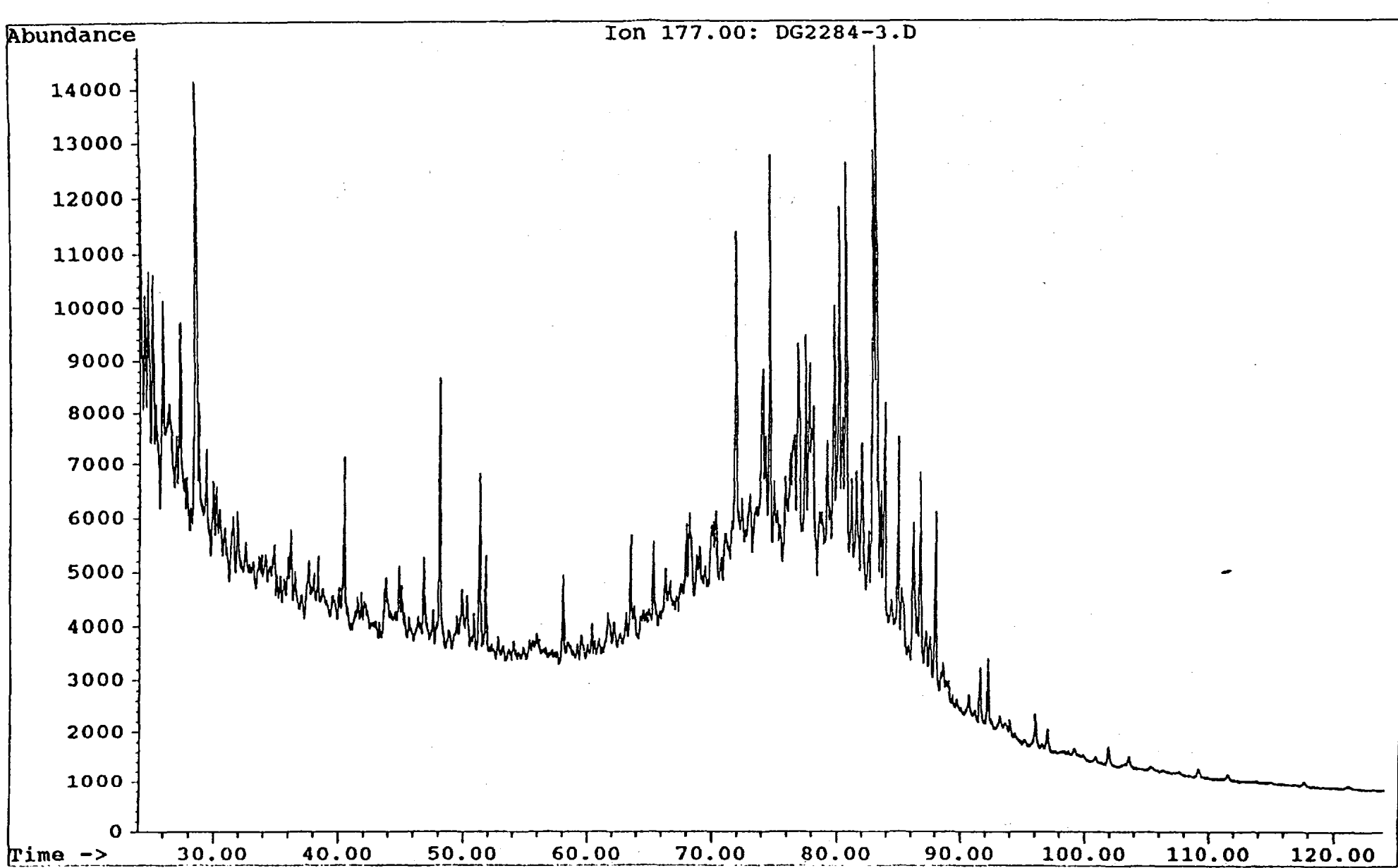


TA3

\begin{tabular}{|c|c|c|}
\hline \multicolumn{2}{|c|}{ Peak ID. } & Sterane Name \\
\hline 1 & A & C27 ba diasterane (20S) \\
\hline 2 & $\mathbf{B}$ & C27 ba diasterane (20R) \\
\hline 3 & C & C27 ab diasterane (20S) \\
\hline 4 & D & C27 ab diasterane (20R) \\
\hline 5 & $\varepsilon$ & C28 ba diasterane (20S) \\
\hline 6 & $F$ & C28 ba diasterane $(20 R)$ \\
\hline 7 & G & $\mathrm{C} 27$ aaa $(20 S)+C 28$ ab dia-(20S) \\
\hline 8 & $\mathrm{H}$ & $C 27$ abb $(20 R)+C 29$ ba dia-(20S) \\
\hline 9 & 1 & $C 27 a b b(20 S)+C 28 a b$ dia-(20R) \\
\hline 10 & J & C27 aaa sterane (20R) \\
\hline 11 & K & C29 ba diasterane (20R) \\
\hline 12 & $L$ & C29 ab diasterane (20S) \\
\hline 13 & $M$ & C28 aaa sterane (20S) \\
\hline 14 & $N$ & C28 abb sterane (20R) \\
\hline 15 & 0 & C28 abb sterane (20S) \\
\hline 16 & $P$ & C28 aaa sterane (20R) \\
\hline 17 & Q & C29 aaa sterane (20S) \\
\hline 18 & $\mathbf{R}$. & C29 abb sterane (20R) \\
\hline 19 & S & C29 abb sterane (20S) \\
\hline 20 & $T$ & C29 aaa sterane (20R) \\
\hline
\end{tabular}

\begin{tabular}{rrc} 
Ret Time & \multicolumn{1}{c}{ Area } & Area\% \\
63.463 & 554245 & 5.74 \\
65.301 & 349024 & 3.62 \\
66.686 & 254904 & 2.64 \\
67.636 & 217157 & 2.25 \\
68.13 & 932107 & 9.66 \\
70.054 & 247728 & 2.57 \\
71.437 & 192717 & 2.00 \\
71.826 & 1826287 & 18.92 \\
72.209 & 369222 & 3.83 \\
73.409 & 146239 & 1.52 \\
73.943 & 1332036 & 13.80 \\
74.861 & 579387 & 6.00 \\
76.093 & 165180 & 1.71 \\
76.734 & 176720 & 1.83 \\
77.098 & 290761 & 3.01 \\
78.542 & 195661 & 2.03 \\
79.885 & 538162 & 5.58 \\
80.673 & 478387 & 4.96 \\
80.924 & 454797 & 4.71 \\
82.664 & 350724 & 3.63
\end{tabular}




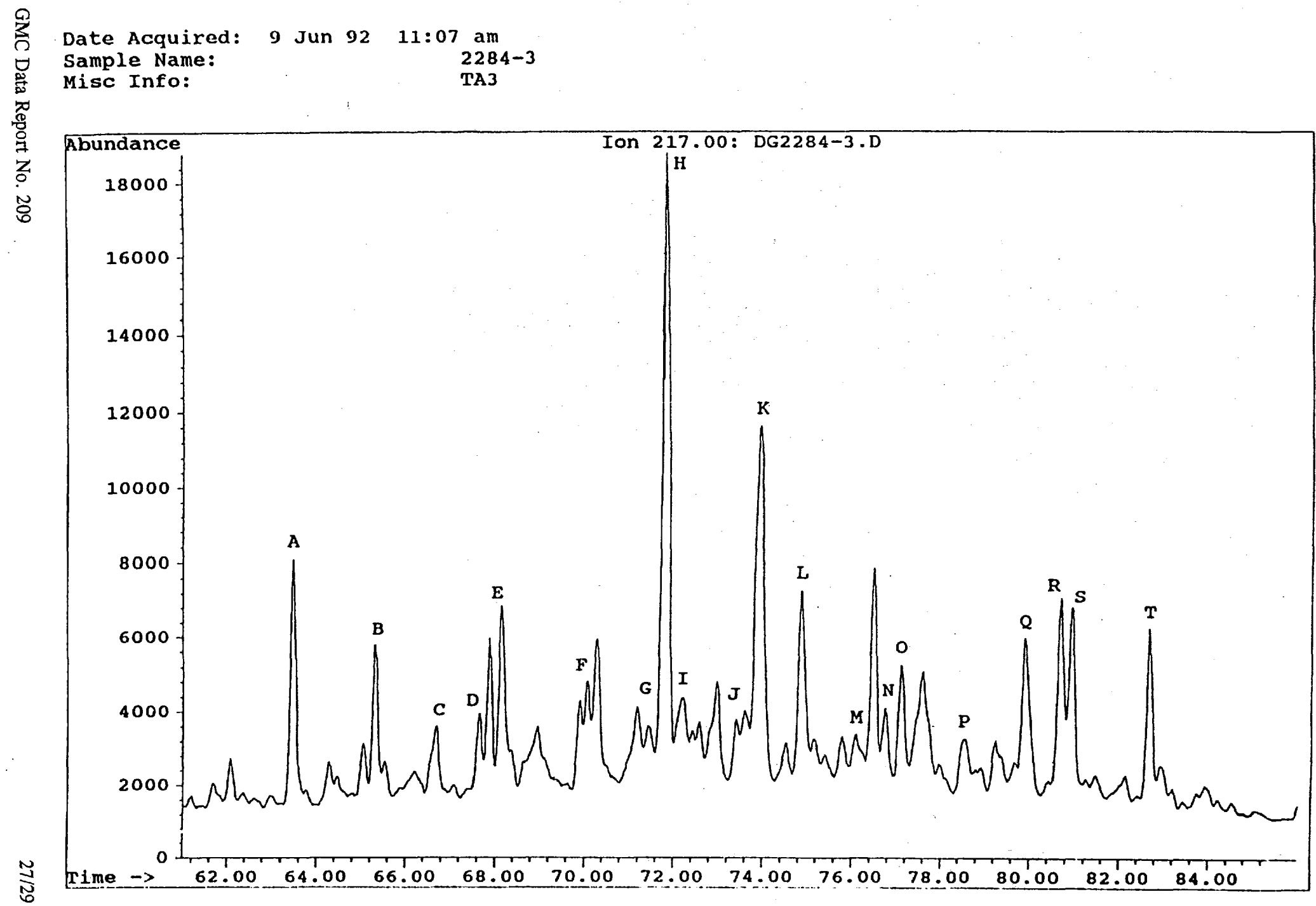




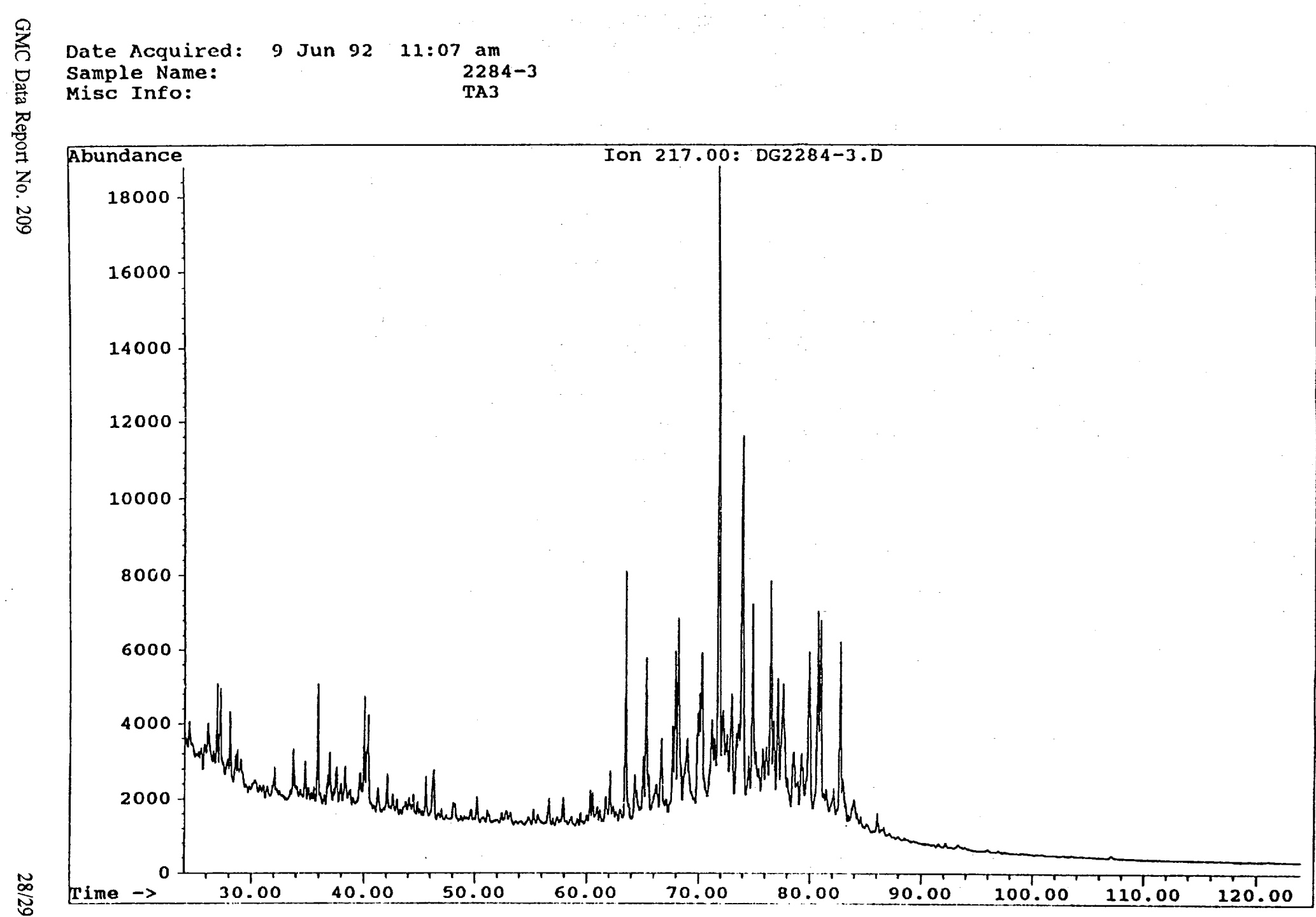


Date Acquired: 9 Jun 92 11:07 am

Sample Name: 2284-3

Misc Info: TA3

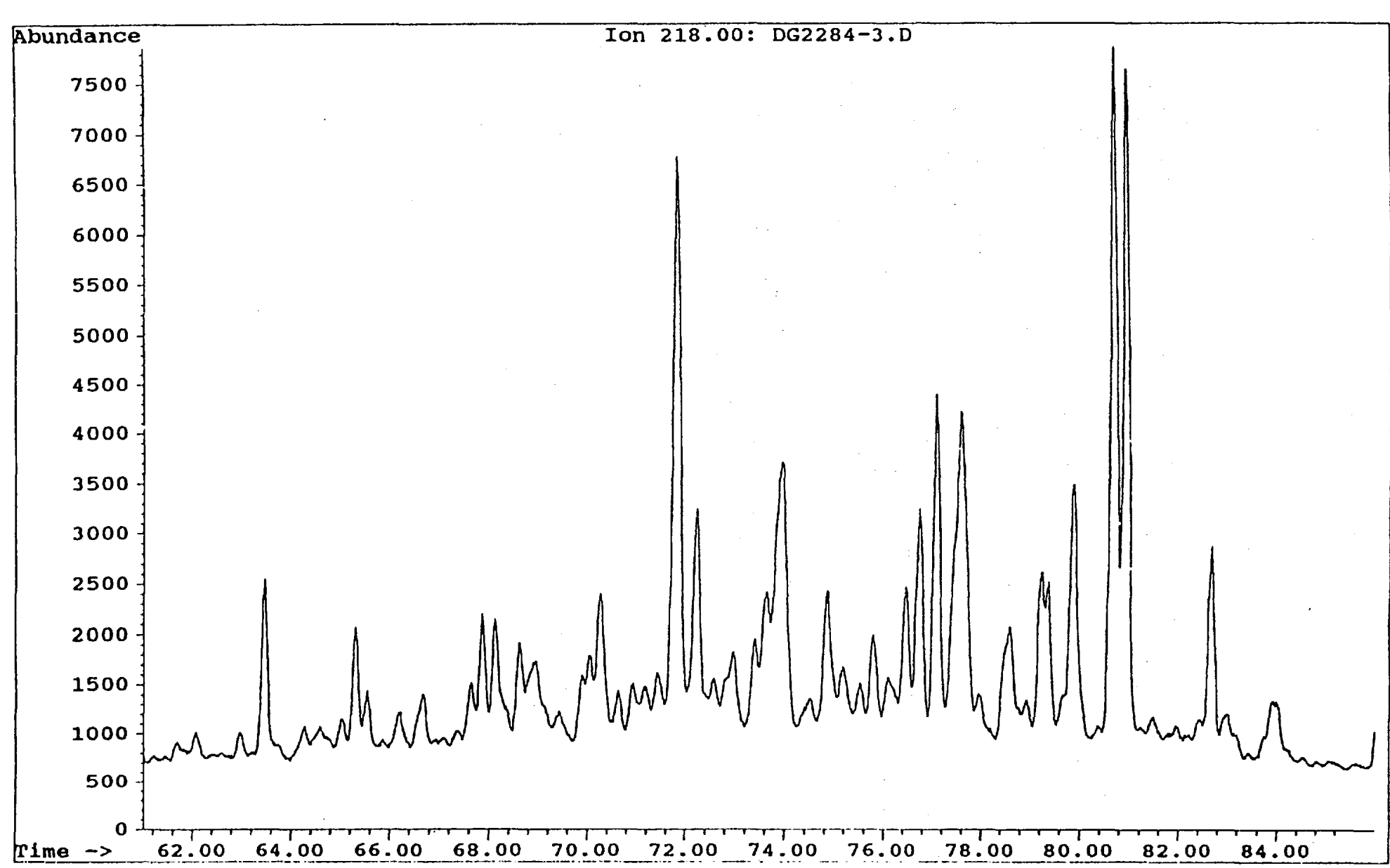

